\title{
Analysis and Forecasts for Trends of COVID-19 in Pakistan using Bayesian Models
}

\author{
Navid Feroze ${ }^{\text {Corresp., } 1}$, Kamran Abbas $^{1}$, Farzana Noor $^{2}{ }^{2}$ Amjid Ali $^{3}$ \\ 1 Department of Statistics, The University of Azad Jammu and Kashmir, Muzaffarabad, Pakistan \\ 2 Department of Mathematics and Statistics, International Islamic University, Islamabad, Pakistan \\ 3 Department of Statistics, Islamia College, Peshawar, Khyber Pakhtunkhwa, Pakistan \\ Corresponding Author: Navid Feroze \\ Email address: navidferoz@gmail.com
}

Background. The COVID-19 is currently on full flow in Pakistan. Given the health facilities in the country, there are serious threats that upcoming moths can be very testing for all the stakeholders. Therefore, there is a need to analyze and forecast the trends of COVID-19 in Pakistan. Methods. We have analyzed and forecasted the patterns of this pandemic in the country, for next thirty days, using Bayesian structural time series models. The causal impacts of lifting lockdown have also been investigated using intervention analysis under Bayesian structural time series models. The forecasting accuracy of the proposed models has been compared with frequently used autoregressive integrated moving average models. The validity of the proposed model has been investigated using similar datasets from neighboring countries including Iran and India. Results. We observed the improved forecasting accuracy of Bayesian structural time series models as compared to frequently used autoregressive integrated moving average models. As far as the forecasts are concerned, on August 10, 2020, the country is expected to have 333,308 positive cases with $95 \%$ prediction interval $[275,034 ; 391,077]$. Similarly, the number of deaths in the country is expected to reach $7,187[5,978 ; 8,390]$ and recoveries may grow to $279,602[208,420 ; 295,740]$. The lifting of lockdown has caused an absolute increase of 98,768 confirmed cases with $95 \%$ interval [85,544; $111,018]$, during the post-lockdown period. The positive aspect of the forecasts is that the number of active cases is expected to decrease to 63,706 [18,614; 95,337], on August 10, 2020. This is the time for the concerned authorities to further restrict the active cases so that the recession of the outbreak continues in the next month. Keywords: BSTS models, lockdown, Ljung Box test, ARIMA models 


\section{Analysis and Forecasts for Trends of COVID-19 in 2 Pakistan using Bayesian Models}

${ }^{1}$ Department of Statistics, The University of Azad Jammu and Kashmir, Muzaffarabad, Pakistan

${ }^{2}$ Department of Mathematical and Statistics, International Islamic University, Islamabad, Pakistan.

Corresponding Author

Department of Statistics, The University of Azad Jammu and Kashmir, Muzaffarabad, Pakistan Email address: navidferoz@gmail.com

\section{Abstract}

Background. The COVID-19 is currently on full flow in Pakistan. Given the health facilities in the country, there are serious threats that upcoming moths can be very testing for all the stakeholders. Therefore, there is a need to analyze and forecast the trends of COVID-19 in Pakistan.

Methods. We have analyzed and forecasted the patterns of this pandemic in the country, for next thirty days, using Bayesian structural time series models. The causal impacts of lifting lockdown have also been investigated using intervention analysis under Bayesian structural time series models. The forecasting accuracy of the proposed models has been compared with frequently used autoregressive integrated moving average models. The validity of the proposed model has been investigated using similar datasets from neighboring countries including Iran and India.

Results. We observed the improved forecasting accuracy of Bayesian structural time series models as compared to frequently used autoregressive integrated moving average models. As far as the forecasts are concerned, on August 10, 2020, the country is expected to have 333,308 positive cases with $95 \%$ prediction interval $[275,034 ; 391,077]$. Similarly, the number of deaths in the country is expected to reach $7,187[5,978 ; 8,390]$ and recoveries may grow to 279,602 $[208,420 ; 295,740]$. The lifting of lockdown has caused an absolute increase of 98,768 confirmed cases with $95 \%$ interval $[85,544 ; 111,018]$, during post-lockdown period. The positive aspect of these forecasts is that active cases are expected to decrease to 63,706 [18,614; 95,337], on August 10, 2020. This is the time for the concerned authorities to further restrict the active cases so that the recession of the outbreak continues in the next month.

Keywords: BSTS models, lockdown, Ljung Box test, ARIMA models 
40

41

42

43

44

45

46

47

48

49

50

51

52

53

54

55

56

57

58

59

60

61

62

63

64

65

66

67

68

69

70

71

72

73

74

75

76

77

78

79

\section{Introduction}

During December, 2019, the historical pandemic started in Wuhan, China (Paules et al., 2020). This pandemic was named novel corona virus or COVID-19 by the World Health Organization (WHO, 2020). Though this virus has lower death rates as compared to Middle East respiratory syndrome (MERS) and severe acute respiratory syndrome (SARS), this virus has higher transmission rates (Tomar and Gupta, 2020). Due to higher transmissibility, the virus has covered almost the whole world (WHO, 2020). In Pakistan, the first positive case was observed on February 26, 2020. The country imposed complete lockdown on March 23, 2020. However, the struggling economy of the country forced the government to lift the lockdown on May 9, 2020. During the lockdown, the number of new cases was low (Yousaf, et al., 2020), however the outbreak has increased pace since lifting of the lockdown. And there are further expectations that number of cases and deaths will increase more rapidly in future (Yousaf, et al., 2020).

The repeated short term forecasts for the patterns of the pandemic are fundamental (Li et al., 2020; Wang and Zhang, 2020 and Zhou et al., 2020). These short term forecasts help the policy makers to make informed decisions in accordance with the evolving scenarios (Ippolito et al., 2020 and McCloskey et al., 2020). There are number of contributions regarding the short terms forecasts for the various parameters of the pandemic in different countries. Some important studies have been conducted in China (Li et al., 2020; Perc et al., 2020 and Fanelli and Piazza, 2020), France (Fanelli and Piazza, 2020), Germany (Perc et al., 2020), India (Tomar and Gupta, 2020 and Gupta and Pal, 2020), Iran (Perc et al., 2020, Zhan et al., 2020 and Moftakhar et al., 2020), Italy (Fanelli and Piazza, 2020; Zhan et al., 2020), Nigeria (Majeed et al., 2020), South Korea (Zhan et al., 2020) and United States (Perc et al., 2020). The literature contains lots of models to obtain forecasts/predictions in different situations; machine learning (Qu, 2016; Le, 2019 and Liu et al., 2020), deep learning (Le et al., 2017 and Le et al., 2019), ARIMA models (Majeed et al., 2020; Benvenuto et al., 2020; Zhang et al., 2020) and Neural Network (Moftakhar et al., 2020).

There has been an earlier attempt to forecast the number of cases, recoveries and deaths in Pakistan (Yousaf, et al., 2020 and Khan et al., 2020). However, the contribution by Yousaf, et al. (2020) was based on quite smaller datasets which is serious issue for producing reliable predictions (Moftakhar et al., 2020). Furthermore, these forecasts underestimated the number of confirmed cases and deaths which may be due to changing post-lockdown trends in the country. On the other hand, the contribution by Khan et al. (2020) did not considered the causal impacts of the lockdown in the country. In addition, the said contributions used the ARIMA models (Yousaf, et al., 2020) and vector autoregressive models (Khan et al., 2020) for the forecasts. The ARIMA models have been frequently used to forecast the patterns of this pandemic (Gupta and Pal, 2020; Majeed et al., 2020; Benvenuto et al., 2020 and Kumar et al., 2020). However, these models have some limitations. Firstly, the forecasts from these models are dependent on the 
previous behavior of the data along with preceding forecast errors, which means the forecasting error accumulates over time. In addition, the forecasting accuracy of these models is affected in presence of covariates (Brockwell and Davis, 2002). To avoid such issues, the Bayesian structural time series (BSTS) models are employed (Scott and Varian, 2013 and Brodersen et al. 2015). The BSTS models (i) allow the inclusion of prior information (ii) allow the model parameters to evolve over time (iii) can handle large number of covariates using spike and slab prior (iv) are less dependent on certain hypothesized specifications (v) can investigate individual components of a time series (McQuire et a.1, 2019). Interestingly, number of time series models, such as ARIMA (used by Yousaf, et al., 2020) and vector autoregressive models (used by Khan et al., 2020), can be expressed in the state space form (Scott, and Varian, 2014b). These models have the capability to provide reliable forecasts for future outbreak of different diseases (Scott and Varian, 2014a). The said models have been used earlier in forecasting the health issues by using alcohol and alcohol licensing policies (de Vocht et al., 2017; McQuire et al., 2019). The proposed models provided $14 \%$ improvement (while comparing with ARIMA models) in forecasting the consumer sentiments (Scott and Varian, 2014a).

The strict social distancing in Wuhan facilitated China to restrict the spread of the pandemic in other provinces ( $\mathrm{Li}$ et al., 2020). However, in Pakistan the lockdown was lifted at quite earlier stage of the pandemic. Unfortunately, to the best of our knowledge, the effects of ending the lockdown has not been studied yet. Therefore, the analysis of the impacts of lifting the lockdown in the country is very important. The causal impacts of different interventions can also be computed using BSTS models. Unlike ARIMA models, these models compute the dynamic confidence interval for the evolving impact, based on difference between actual and counterfactual series. Due to their added advantages, these models are superior to conventional models (Brodersen et al., 2015).

We have conducted a study to investigate the various parameters of COVID-19 in Pakistan, for next thirty days. Since the Bayesian methods often produce better results as compared classical methods (Kundu and Joarder, 2006; Kundu, 2008 and Park et al. 2013), the BSTS models have been proposed for the forecasting. The causal impacts of lifting the lockdown in the country have also been investigated conducting intervention analysis within BSTS models. The improved forecast accuracy has been observed using BSTS models, instead of repeatedly used ARIMA models. We have also included the datasets from neighboring countries (India and Iran) to investigate the performance of the proposed models for data regarding other countries. The detailed study suggested that the cumulative number of confirmed cases and deaths is expected to increase exponentially during next month. However, the recoveries are expected to increase faster than confirmed cases, so the active number of cases will decrease during the next month. The study also revealed that lifting the lockdown at the earlier stage has seriously increased the trajectory of the outbreak in the country. 
120

121

122

123

124

125

126

127

128

129

130

131

132

133

134

135

136

137

138

139

140

141

142

143

144

145

146

147

148

149

150

151

152

153

154

155

156

\section{Materials \& Methods}

The data have been obtained from the published reports of the National Institute of Health (NIH), Islamabad, Pakistan. The data contain the details regarding cumulative (and daily) number of cases, deaths, recoveries and tests in the country (NIH, 2020). NIH updates the data on daily basis since February 26, 2020. Additionally, data for Iran and India are obtained from reports of Our World in Data (Our World in Data, 2020). As we have used the published data by NIH and Our World in Data, ethical approval was not required.

\section{ARIMA Models}

The ARIMA model is mathematically written as $\operatorname{ARIMA}(\mathrm{p}, \mathrm{d}, \mathrm{q})$. The model is based on three parameters ' $p, d$ and $q$ '. The parameter ' $p$ ' represents the autoregressive terms, ' $d$ ' defines the number of non-seasonal differences and ' $q$ ' denotes the number of moving terms. The $\operatorname{ARIMA}(\mathrm{p}, \mathrm{d}, \mathrm{q})$ model can be written as

$$
Z_{t}=\theta_{1} z_{t-1}+\theta_{2} z_{t-2}+\ldots+\theta_{p} z_{t-p}+\varepsilon_{1}-\lambda_{1} \varepsilon_{t-1}-\varepsilon_{2}-\lambda_{2} \varepsilon_{t-2}-\ldots-\varepsilon_{q}-\lambda_{q} \varepsilon_{t-q}
$$

where, $\theta_{p}$ represents the terms of autoregressive operator, $\varepsilon_{q}$ are the coefficients of the error terms, $\lambda_{q}$ are the values of moving average operator and $Z_{t}$ is d-order differenced time series.

\section{Structural Time Series Models}

Visualization of a time series as a product of aggregating different components is very useful. The decomposition of each layer facilitates the direct individual interpretation of the model. The structural time series models have these features. The basic form of the structural time series models (STM) is as follows

$Y_{t}=T_{t}+S_{t}+E_{t}$; where $Y_{t}$ is the observed value, $T_{t}$ represents the trend component, $S_{t}$ denotes the seasonal component, and $E_{t}$ is the error term. Hence, the STM is a dynamic system composed of trend and seasonal components perturbed by random errors. The STM is capable of reflecting the important characteristics of the data and enhancing prediction power of the model by including repressors. These models also utilize the previous knowledge about the parameters by adding the prior information.

The Gaussian STM can be expressed as

$Y_{t}=W_{t} \lambda_{t}+\omega_{t} ; \omega_{t}: N\left(0, \Phi_{t}\right)$

$\lambda_{t+1}=X_{t} \lambda_{t}+H_{t} \mu_{t} ; \mu_{t}: N\left(0, \Omega_{t}\right)$

Equation (2) links the observed data $Y_{t}$ with the unobserved latent variable $\lambda_{t}$, hence called observation equation. In (2), $Y_{t}$ is $k \times 1$ vector of values, $W_{t}$ is a $k \times m$ matrix involving known values, $\lambda_{t}$ is unobserved $k \times 1$ state vector and $\omega_{t}$ are the randomly and independently 
157 distributed Gaussian error terms with zero mean and variance $\Phi_{t}$. On the other hand, Equation

158 (3) is called transition equation as it defines the behavior of latent state over time. It is simply an 159 autoregressive model of $\lambda_{t}$, defined by the unobservable Markov Chain process observed by $Y_{t}$. 160 Here, the $X_{t}$ is $k \times k$ transition matrix, $H_{t}$ is $k \times m$ error control matrix (indentifies the rows of 161 transition equation having non-zero error terms), and $\mu_{t}$ another Gaussian random error term 162 with mean zero and variance $\Omega_{t}$. Therefore, the STM contain the underlying stochastic process 163 determined by $\lambda_{t}$. Since $\lambda_{t}$ are unobservable, a vector of observations is used to compute the 164 system. The initial information in shape of prior information is assumed for $\lambda$ which is normally 165 distributed with mean $\lambda_{t}$ and variance $Q_{0}$ which is independent of $\omega_{t}$ and $\mu_{t}$ for all t.

166

Suppose $\beta$ is a set of all model parameters and $\alpha=\left(\alpha_{1}, \alpha_{2}, \ldots, \alpha_{m}\right)$ represents the full state sequence. Further suppose that the prior distribution for $\beta$ is $\pi(\beta)$ as well as distribution $\pi\left(\alpha_{0} \mid \beta\right)$ on the initial state values. Then, the sample from the posterior distribution $p(\alpha, \beta \mid \boldsymbol{Y})$ can be obtained using MCMC method in the following manner. Draw a sequence of $\left(\alpha_{1}, \beta_{1}\right),\left(\alpha_{1}, \beta_{1}\right), \ldots$ from a Markov chain having stationary distribution as $p\left(\alpha, \beta \mid \boldsymbol{Y}_{1: n}\right)$. The

174

175

176

177

178

179

180

181

182

183

184

185

186

187

188

189

190

191

192

\section{Markov Chain Monte Carlo (MCMC) Method}

sampler alternates between the data augmentation step and parameter simulation step. The data augmentation step and parameter estimation step simulate from conditional distributions $p\left(\alpha \mid \boldsymbol{Y}_{1: n}, \beta\right)$ and $p\left(\beta \mid \boldsymbol{Y}_{1: n}, \alpha\right)$, respectively. The data augmentation step has been carried out using the algorithm proposed by Durbin and Koopman (2002) which provides the improvement in the algorithm proposed by De Jong and Shephard (1995). Once the draws for the state are available, the draws for the parameters are easy to obtain for all state components excluding the static regression coefficients. The draws for the static regression coefficients have been obtained using the algorithm proposed by Ghosh and Clyde (2011). The detailed studies regarding implementation of MCMC algorithms can be seen from the contributions of (Fitzgerald, 2001; Bugallo et al., 2015; and Martino, 2018).

\section{Causal Impacts}

Equations (2-3) also investigate the post-lockdown discrepancy between observed number of new cases, deaths and active cases and a simulation based series that was expected without lifting the lockdown. Such investigations provide facilitations in assessing the causal impacts of lifting lockdown in the country. These causal impacts can be computed by the following steps.

Step-I: $\quad$ Fit STM using observations under the lockdown period.

Step-II: $\quad$ Use fitted model from Step-I to produce forecasts for post-lockdown period. 
193

194

195

196

197

198

199

200

201

202

203

204

205

206

207

208

209

210

211

212

213

214

215

216

217

218

219

220

221

222

223

224

225

226

227

228

229

230

231

Step-III: $\quad$ The difference between forecasted series and actual series during the postlockdown period is computed and interpreted as causal impacts of lifting the lockdown in the country.

The causal impact model incorporates the behavior of two time series (i) the behavior of the response series in the pre-intervention period (ii) the behavior of a time series that were predictive of the response series in the pre-intervention period. If the control series did not encountered the intervention, it can be assumed that the relationship that existed between the treatment and control in the pre-intervention period may continue in the post-intervention period (Brodersen et al., 2015). We used number tests as the control series, which were predictive of the number of cases/deaths prior to the lifting of the lockdown. The number of tests was itself not affected by the lifting of the lockdown; hence the assumptions for the implementation of the causal impact model were not affected.

We have used $\mathrm{R}$ software to conduct the analysis regarding the study. The computations for ARIMA models have been obtained using auto.arima function available in forecast package, while bsts package has been used to obtain the forecasts for the BSTS models (Scott, 2020). We have employed the local level model for the trend component. The number seasons have been chosen to be seven to evaluate the weekly pattern of the series. The root mean square percentage error (RMSPE) and root median square percentage error (RMdSPE) have been used to compare the forecast accuracy of proposed models with ARIMA models. The said measures can efficiently determine the forecast accuracy of a model (Hyndman and Koehler, 2006 and Bowerman et al., 2004). Based on these measures, we have investigated the predictive power of BSTS models using different starting points. To investigate whether the residuals are white noise, we used Ljung Box test at different lags. We have also included the datasets from neighboring countries (India and Iran) to observe the applicability of the proposed models in different situations. As we observed the improved forecasting capacity of BSTS models, the detailed forecasts have only been reported under BSTS models for brevity. The forecasts under BSTS models are based on prior information and current data (likelihood function). The bsts package uses spike-slab prior for the analysis. The prior information is combined with the likelihood function to produce the posterior distribution. The BSTS models often use the default prior. The default prior for the variance $\left(\sigma^{2}\right)$ can be formulated as: $\sigma^{-2}: G\left(10^{-2}, 10^{-2} s_{Y}^{2}\right)$, where $G(a, b)$ is gamma distribution with parameters ' $a$ ' and 'b' and $s_{Y}^{2}=\sum_{t}\left(Y_{t}-\bar{Y}\right)^{2} /(n-1)$. On the other hand in case of several controls, the spike and slab prior is used for the coefficients (Scott and Varian, 2014b). The prior parameters in spike and slab are selected using Zellner's prior method (Liang et al., 2008; Zellner, 1986). The posterior distribution is estimated using Gibbs sampler (George and Mcculloch, 1997). The detailed studies regarding implementation of MCMC algorithms can be seen from the contributions of (Fitzgerald, 2001; Bugallo et al., 2015; and Martino, 2018). In addition the Kalman filter and Bayesian model averaging were employed 
232 to obtain the forecasts. The causal impacts of lifting the lockdown are investigated by conducting

233 intervention analysis within the BSTS models. The CausalImpact package in R (Brodersen and 234 Hauser, 2020), has been used for estimating the said impacts.

235

236

237

238

239

240

241

242

243

244

245

246

247

248

249

250

251

252

253

254

255

256

257

258

259

260

261

262

263

264

265

266

267

268

269

270

271

\section{Results \& Discussions}

On July 11, 2020, Pakistan had 246,351 number of total confirmed cases, 93,217 active cases, 5123 deaths and 153,134 recoveries regarding COVID-19. The ratio of cumulative confirmed cases per 100 tests (RCT) stood at 16.01. Similarly, the ratio of cumulative deaths per 100 confirmed cases (RDC) was 2.08, and the ratio of cumulative number of recoveries per 100 confirmed cases (RRC) was 62.12. These figures have already challenged the healthcare system of Pakistan having total number of 215,436 doctors, 108,137 nurses, 19,218 community health workers (CHWs) and 41,689 labs across the country (WHO, 2020). The government figures also suggest that there are 6664 crucial care beds and about 2500 Ventilators for the COVID-19 patients in the country $(\mathrm{NIH}, 2020)$. These figures simply indicate that Pakistan has very limited healthcare infrastructure to cope up this pandemic. Hence, analysis of different parameters of this epidemic is fundamental for watchful decision making in the country. Using the dataset obtained from NIH, we compared the forecasting accuracy of BSTS models with ARIMA models. The comparison is made on the basis of RMSPE and RMdSPE. The current trends of the pandemic have been represented in Figure 1. The results regarding comparison of predictive powers of the proposed models are given in Table 1. The diagnostic checking for the proposed models, using Ljung Box test, has been reported in Table 2. The future trends of the outbreak are presented in Table 3 and in Figure 2. The separated analysis of trend, seasonality and regression components has been presented in Figure 3. On the other hand, the causal impact of lifting lockdown in the country has been discussed in Figure 4.

Figure 1 reports the current trajectories of COVID-19 in Pakistan, where the zeroth day denotes February 25, 2020. In particular, this figure includes the trends of RCT, RDC, RRC and RAC. Though RAC has decreasing trend and RRC has increasing trend, the issue for the country is that $\mathrm{RDC}$ is constant over time, which means that rate of deaths is directly proportional to number of cases. In addition, the RCT has increased over time, which is quit alarming. It means the relative pace of spread has increased over time. The comparison of forecast accuracy for ARIMA and BSTS models are placed in Table 1. The numerical results for RMSPE and RMdSPE for each country (Pakistan, Iran and India) have been reported in the said Table. We have used different starting points to check the predictive power of the proposed models. In particular we have used four different starting points which include $90 \%, 80 \%, 70 \%$ and $60 \%$ of the original series. For each staring point the values of RMSPE and RMdSPE are less than $10 \%(0.10)$, which indicates the predictive power of the proposed model is good enough. The results in this table also suggest the amounts for RMSPE and RMdSPE are smaller for BSTS models, with few exceptions. Hence, the forecast accuracy of BSTS models is better than that of ARIMA models. The trace

Peer] reviewing PDF | (2020:07:50949:2:1:NEW 1 May 2021) 
272 plots for the one-step-ahead prediction error variance (PEV), from the MCMC chains, have also

273

274

275

276

277

278

279

280

281

282

283

284

285

286

287

288

289

290

291

292

293

294

295

296

297

298

299

300

301

302

303

304

305

306

307

308

309

310 been used for the evaluation of the prediction power of the proposed model. The Figures 5-8 represent the trace plots for PEV regarding cumulative number of cases, deaths, recoveries and active cases in Pakistan, respectively. Each of the said figures suggested the good convergence of the estimates. Further, QQ plots for the residual terms have been given in Figures 9-12. In QQ plots, the black line is the reference line for the standard normal distribution and means of MCMC draws have been represented by blue dots. Since the dots are clustered around the straight line, the normality assumption holds well for the residuals. In additions, we have also plotted the posterior distribution of the autocorrelation function (ACF) of residuals using boxplots, for Pakistan, in Figures 13-16. Since the ACF plots have dampened out at successive lags, so the residual terms are stationary for each variable. Further, the numerical results regarding diagnostic checking of the proposed models have been presented in Table 2. From these results, it can be seen that $\mathrm{P}$-values for the Ljung Box test(Q-statistic) are greater than 0.05 for all variables at different lags. So, we do not have sufficient evidence to assume the residuals as dependent. The calibration for the models using 90\%, 80\%, 70\%, 60\% and $50 \%$ data has been reported in Figures 17-21, respectively. From the said figures, it can be seen that actual data are within the corresponding $95 \%$ prediction intervals.

Hence, the models can efficiently be used to derive the forecast for the epidemic. Table 3 and Figure 2 represent the forecasts for various parameters of the epidemic using BSTS models. It should be noted that this figure has logarithmic scale for y-axis, so the straight line indicate the exponential growth. From this figure, it can be assessed that estimated series is quite close to the observed data (mostly overlapping the observed series), which indicates the model efficiency. Further, the cumulative number of positive cases, recoveries and deaths is expected to increase exponentially during the next thirty days. However, the growth in number of recoveries is expected to be faster than that of confirmed case, which is a good sign for the country. To be more specific, on August 10, 2020, the expected number of positive cases in Pakistan will be 333,308 with $95 \%$ prediction interval $[275,034 ; 391,077]$. Similarly, the number of deaths in the country is expected to reach $7,187[5,978 ; 8,390]$ and recoveries may grow to $279,602[208,420$; 295,740]. However, it was very encouraging to observe that the active cases are likely to decrease to 63,706 [17,614; 95,337], which is lower as compared to current number. This relief is mainly due to rapid growth in the recoveries in the country. The demand for the healthcare facilities is expected to be slightly lower in the next month. The health administration should utilize this opportunity to improve the quality of health services regarding the COVID-19. On the other hand, Iran is expecting 325,391 [283,403; 369,613] active cases, 17,458 [15,538; 19,476] deaths, 294,493 [243,470; 350,203] recoveries and 5,892 [973; 73,416] active cases on August 10,2020 . Hence, Iran is expecting quite comprehensive control over the pandemic in the next thirty days. However, the situation of the pandemic in India can be quite serious during next month. Our forecasts for India suggest that the country should expect 1,874,037 [1,538,452;

Peer] reviewing PDF | (2020:07:50949:2:1:NEW 1 May 2021) 
311

312

313

314

315

316

317

318

319

320

321

322

323

324

325

326

327

328

329

330

331

332

333

334

335

336

337

338

339

340

341

342

343

344

345

346

347

348

349

350

2,393,316] active cases, 39,464 [30,407; 48,765] deaths, 1,299,328 [1,091,762; 1,618,996] recoveries and 517,153 [390,286; 717,715] active cases up to the target date (August 10, 2020).

The BSTS models also allow us to investigate the patterns of trend, seasonality and regressions individually. We have investigated the contribution of these components for cumulative number of positive cases, as the patterns for the other parameters were alike. As the cumulative number of positive cases depends on the cumulative number of tests, we considered the cumulative number of tests conducted in the country as covariate. The contributions of the said components have been presented in Figure 3. This figure elucidates that the cumulative number of positive cases is increasing exponentially in the country. The contribution of seasonality is slightly increasing over time. In addition, the contribution of regression component is also has an upward trend, this is mainly due to increase in rate of confirmed cases.

The causal impact of lifting the lockdown in the country has also been discussed by conducting intervention analysis using BSTS models. The results have been given in Figure 4. This figure simply indicates the significant increase in pace of the outbreak (in black lines) as compared to expected, had the lockdown not lifted (in grey shade). The probability of causal impact was 0.9989, which was quite high indicating that the lifting the lockdown has increased the outbreack of the epidemic significantly. On July 11, 2020, the total number of confirmed cases was 246,351; however under lockdown the number of cases would have been 147,583 with $95 \%$ interval $[135,333 ; 160,807]$. So, there is an absolute increase of 98,768 confirmed cases with $95 \%$ interval $[85,544 ; 111,018]$. Hence, the country has conceded $56 \%$ increase (in the confirmed cases) than the expected, if the lockdown would have continued. In addition, during the lockdown, the average rate of positive cases per 100 tests was $8.68 \%$, while for the post lockdown period this rate stands at $13.21 \%$. Similarly, the rate of deaths per 100 positive cases, for the lockdown period, was $1.46 \%$ which increased to $2.06 \%$ in the after the lockdown. On the whole, the ending lockdown has significantly increased the load on the Pakistani healthcare system. It is also a truth that the country cannot afford a prolonged lockdown. However, identifying the hotspots and enforcing lockdown in those areas may help. In addition, the serious effort to induce the people to abide by the SOPs in the country is fundamental.

The results from other studies of this nature are quite compatible. For example, the studies conducted by (Li et al., 2020; Majeed et al., 2020; Tomar and Gupta, 2020; Perc et al., 2020; Fanelli and Piazza, 2020; and Moftakhar et al., 2020), also indicated a rapid future growth in the pandemic in respective countries. Our results are also comparable with the earlier study carried out in Pakistan (Yousaf, et al., 2020). However, this study did not cover the impact lockdown in the country. In addition, we have obtained these forecasts using a more flexible model.

Peer] reviewing PDF | (2020:07:50949:2:1:NEW 1 May 2021) 


\section{Conclusions}

352

353

354

355

356

357

358

359

360

361

362

363

364

365

366

367

368

369

370

371

372

373

374

375

376

377

378

379

380

381

382

383

384

385

386

387

388

The study has been conducted to investigate the different parameters of COVID-19 in Pakistan. The study suggests that the lifting of lockdown has increased the speed of the pandemic in the country. The number of positive cases is directly proportional to the number of tests carried out in the country. The total number of positive cases and deaths is expected to grow exponentially. However, the trajectory of recoveries is likely to be higher than that for confirmed cases. Therefore, the expected number of active cases is likely to decrease in the next month. We suggest that this is the right time where concerned authorities may put more efforts to further minimize the active patients in the country. If it happened, there are expectations that country will see the contraction in the outbreak of this pandemic. It should also be noted that Pakistan cannot afford the complete lockdown due to its frail economy; hence we cannot suggest the complete lockdown in the country again. However, properly identifying hotspots and enforcing lockdown partially may help. The government also needs to follow a strict tracking, tracing and quarantine strategy. And more importantly, all possible efforts and resources should be mobilized to improve the healthcare facilities to meet the needs of the coming month. Similarly, the overall situation of the pandemic in Iran is expected to be quite in control in the next month. However, India is expecting a drastic increase in number of patients, deaths and active cases in the coming month. Though, the number of recovered patients is expected to grow exponentially, the impact of this growth will be seized by the fast growing cases and deaths.

Our study has some limitations too. Firstly, the data may be underreported, as the sufficient random testing has not been considered in the country, and not all the patients report themselves to health officials with a fear of staying fourteen days in quarantine. Secondly, the forecasts are based on the assumption that the current trends will follow in the next month, the forecasts can be misleading if this assumption is violated. Thirdly, the study lacks in investigating the risk factors as the data regarding the demography and social networks of the patients were not available.

\section{References}

Benvenuto, D., Giovanetti, M., Vassallo, L., Angeletti, S. and Picozzi, S. 2020. Application of the ARIMA model on the COVID-2019 epidemic dataset, Data in Brief, 29, 105340. https://doi.org/10.1016/j.dib.2020.105340.

Bowerman, B. L., O’Connell, R. T. and Koehler, A. B. 2004. Forecasting, time series and regression: An applied approach. Belmont, CA7 Thomson Brooks/Cole. p.18.

Brockwell, P. J. and Davis R. A. 2002. Introduction to Time Series and Forecasting, 39, New York; NY: Springer.

Peer) reviewing PDF | (2020:07:50949:2:1:NEW 1 May 2021) 
389

390

391

392

393

394

395

396

397

398

399

400

401

402

403

404

405

406

407

408

409

410

411

412

413

414

415

416

417

418

419

420

421

422

423

424

425

426

427

Brodersen, K. H., Gallusser, F., Koehler, J., Remy, N., \& Scott, S. L. 2015. Inferring causal impact using Bayesian structural time-series models. Annals of Applied Statistics, 9(1), 247274. https:// doi.org/10.1214/14-AOAS788.

Brodersen, K. H. and Hauser, A. 2020. Inferring Causal Effects using Bayesian Structural TimeSeries Models, https://cran.r-project.org/web/packages/CausalImpact.

Bugallo, M. F., Martino, L. and Corander, J. 2015. Adaptive Importance Sampling in Signal Processing, Digital Signal Processing, 47, 36-49.

De Jong, P., \& Shephard, N. 1995. The simulation smoother for time series models. Biometrika, 82(2), 339-350.

de Vocht, F., Tilling, K., Pliakas, T., Angus, C., Egan, M., Brennan, A., Campbell, R. and Hickman, M., 2017. The intervention effect of local alcohol licensing policies on hospital admission and crime: a natural experiment using a novel Bayesian synthetictime-series method. J Epidemiol Community Health, 71(9), 912-918.

Durbin, J., \& Koopman, S. J. 2002. A simple and efficient simulation smoother for state space time series analysis. Biometrika, 89(3), 603-616.

Fanelli, D. and Piazza, F. 2020. Analysis and forecast of COVID-19 spreading in China, Italy and France. Chaos, Solitons \& Fractals, 134, 109761.

Fitzgerald, W. J. 2001. Markov chain Monte Carlo methods with applications to signal processing, Signal Processing, 81(1), 3-18.

Ghosh, J., \& Clyde, M. A. 2011. Rao-blackwellization for bayesian variable selection and model averaging in linear and binary regression: A novel data augmentation approach. Journal of the American Statistical Association, 106(495), 1041-1052.

Gupta, R.. and Pal, S. K. 2020. Trend Analysis and Forecasting of COVID-19 outbreak in India. MedRxiv. https://doi.org/10.1101/2020.03.26.20044511.

Hyndman, R. J. and Koehler, A. B. 2006. Another look at measures of forecast accuracy. International Journal of Forecasting, 22(4), 679-688.

Ippolito, G., Hui, D. S., Ntoumi, F., Maeurer, M. and Zumla, A. 2020. Toning down the 2019nCoV media hype - and restoring hope. Lancet Respir Med. 8:230-1. doi: 10.1016/S22132600(20)30070-9.

Kumar, P., Kalita, H., Patairiya, S., Sharma, Y. D., Nanda, C., Rani, M., Rahmai, J. and Bhagavathula A. S. 2020. Forecasting the dynamics of COVID-19 Pandemic in Top 15 countries in April 2020 through ARIMA Model with Machine Learning Approach. MedRxiv. https://doi.org/10.1101/2020.03.30.20046227.

Le, N. Q. K. 2019. iN6-methylat (5-step): identifying DNA N 6-methyladenine sites in rice genome using continuous bag of nucleobases via Chou's 5-step rule. Molecular Genetics and Genomics, 294(5), 1173-1182. (machine)

Le, N. Q. K., Ho, Q. T., \& Ou, Y. Y. 2017. Incorporating deep learning with convolutional neural networks and position specific scoring matrices for identifying electron transport proteins. Journal of Computational Chemistry, 38(23), 2000-2006.

Peer] reviewing PDF | (2020:07:50949:2:1:NEW 1 May 2021) 
428

429

430

431

432

433

434

435

436

437

438

439

440

441

442

443

444

445

446

447

448

449

450

451

452

453

454

455

456

457

458

459

460

461

462

463

464

465

466

467

Le, N. Q. K., Yapp, E. K. Y., Nagasundaram, N., Chua, M. C. H., \& Yeh, H. Y. 2019. Computational identification of vesicular transport proteins from sequences using deep gated recurrent units architecture. Computational and Structural Biotechnology Journal, 17, 12451254.

Li, Q., Guan, X., Wu, P., Wang, X., Zhou, L., Tong, Y., Ren, R., Leung, K.S., Lau, E.H., Wong, J.Y. and Xing, X., 2020. Early transmission dynamics in Wuhan, China, of novel coronavirus-infected pneumonia. New England Journal of Medicine, 382:1199-207. doi: 10.1056/NEJMoa20 01316.

Liang, F., Paulo, R., Molina, G., Clyde, M. A., \& Berger, J. O. 2008. Mixtures of g priors for Bayesian variable selection. Journal of the American Statistical Association, 103(481), 410423.

Liu, Z., Huang, S., Lu, W., Su, Z., Yin, X., Liang, H., \& Zhang, H. 2020. Modeling the trend of coronavirus disease 2019 and restoration of operational capability of metropolitan medical service in China: a machine learning and mathematical model-based analysis. Global Health Research and Policy, 5, 1-11.

Majeed, K. A., Adeleke, M. Popoola, L. 2020. Online Forecasting of Covid-19 Cases in Nigeria Using Limited Data, Data in Brief, doi: https://doi.org/10.1016/j.dib.2020.105683.

Martino, L. 2018. A Review of Multiple Try MCMC algorithms for Signal Processing, Digital Signal Processing, 75, 134-152.

McCloskey, B., Zumla, A., Ippolito, G., Blumberg, L., Arbon, P., Cicero, A., Endericks, T., Lim, P.L. and Borodina, M., 2020. Mass gathering events and reducing further global spread of COVID-19: a political and public health dilemma. The Lancet, 395(10230), 1096-1099.. doi: 10.1016/S0140-6736(20)30681-4.

McQuire, C., Tilling, K., Hickman, M. and de Vocht, F. 2019. Forecasting the 2021 local burden of population alcoholrelated harms using Bayesian structural time-series, Addiction, 114, 994-1003. DOI:10.1111/add.14568.

Moftakhar, L., Seif, M. and Safe, M. S. 2020. Exponentially Increasing Trend of Infected Patients with COVID-19 in Iran: A Comparison of Neural Network and ARIMA Forecasting Models, Iran J Public Health, 49(1), 92-100.

National Institute of Health (NIH) Islamabad Pakistan. https://www.nih.org.pk/. Accessed July $11,2020$.

Our World in Data (2020). Our World in Data, https://ourworldindata.org/coronavirus-sourcedata, accessed on December 30, 2020.

Paules, C. I., Marston, H. D. and Fauci, A. S. 2020. Coronavirus infections-more than just the common Cold. JAMA, 323(8):707-8.doi:10.1001/ jama.2020.0757.

Perc, M., Gorisek, M. N., Slavinec, M. and Stozer, A. 2020. Forecasting COVID-19. Front. Phys. 8:127. doi:10.3389/fphy.2020.00127. admission committees

Ou, Y. Y. 2016. Prediction of FAD binding sites in electron transport proteins according to efficient radial basis function networks and significant amino acid pairs. BMC bioinformatics, 17(1), 298.

Peer) reviewing PDF | (2020:07:50949:2:1:NEW 1 May 2021) 
468

469

470

471

472

473

474

475

476

477

478

479

480

481

482

483

484

485

486

487

488

489

490

491

492

493

494

495

496

497

498

499

500

Scott, S. L. 2020. Bayesian Structural Time Series, https://www.rdocumentation.org/packages /bsts/versions/0.9.5.

Scott S. L. and Varian H. R. 2014a. Bayesian variable selection for nowcasting economic time series. In: Goldfarb A., Greenstein S. M., Tucker C. E., editors. Economic Analysis of the Digital Economy. Cambridge, MA: National Bureau of Economic Research, Inc; 119-35.

Scott, S. L., \& Varian, H. R. 2014b. Predicting the present with bayesian structural time series. International Journal of Mathematical Modelling and Numerical Optimisation, 5(1-2), 4-23.

Scott, S. L. and Varian, H. R. 2013. Bayesian variable selection for nowcasting economic time series. NBER Working Paper Series. Cambridge. https://doi.org/10.3386/w19567.

Tomar, A. and Gupta, N. 2020. Prediction for the spread of COVID-19 in India and effectiveness of preventive measures, Science of the Total Environment, 728, 138762.

Wang, F. and Zhang, C. 2020. What to do next to control the 2019-nCoV epidemic?. The Lancet 395:10222, 391-393.

World Health Organization (WHO). 2020. Novel coronavirus (2019-nCoV); Available from URL: https:/www.who.int/ emergencies/diseases/novel-coronavirus-2019/situation reports.

Yousaf, M., Zahir, S., Riaz, M., Hussain, S. M. and Shah, K. 2020. Statistical analysis of forecasting COVID-19 for upcoming month in Pakistan, Chaos, Solitons and Fractals, 138, 109926.

Zellner, A. 1986. On assessing prior distributions and Bayesian regression analysis with g-prior distributions. Bayesian inference and decision techniques. Essays in Honor of Bruno de Finetti (P. K. Goel and A. Zellner, eds.). Stud. Bayesian Econometrics Statist, 6, 233-243. North-Holland, Amsterdam. MR0881437 G

Zhan, C., Chi, K. T., Lai, Z., Hao, T. Su, J. 2020. Prediction of COVID-19 Spreading Profiles in South Korea, Italy and Iran by Data-Driven Coding. medRxiv.

Zhang, Y., Yang, H., Cui, H., \& Chen, Q. 2020. Comparison of the ability of ARIMA, WNN and SVM models for drought forecasting in the Sanjiang Plain, China. Natural Resources Research, 29(2), 1447-1464.

Zhou, T., Liu, Q., Yang, Z., Liao, J., Yang, K., Bai, W., Lu, X. and Zhang, W., 2020. Preliminary prediction of the basic reproduction number of the Wuhan novel coronavirus 2019-nCoV. Journal of Evidence-Based Medicine, 13(1), 3-7.. doi: 10.1111/jeb m.12376.

Peer) reviewing PDF | (2020:07:50949:2:1:NEW 1 May 2021) 


\section{Table $\mathbf{1}$ (on next page)}

Measures of forecast accuracy for BSTS models and ARIMA models 


\begin{tabular}{|c|c|c|c|c|c|c|c|c|}
\hline Country/ & \multicolumn{2}{|c|}{ ARIMA } & \multicolumn{2}{|c|}{ BSTS } & \multicolumn{2}{|c|}{ ARIMA } & \multicolumn{2}{|c|}{ BSTS } \\
\hline Parameter & RMSPE & RMdSPE & RMSPE & RMdSPE & RMSPE & RMdSPE & RMSPE & RMdSPE \\
\hline Pakistan & \multicolumn{4}{|c|}{$90 \%$ of the Series } & \multicolumn{4}{|c|}{$80 \%$ of the Series } \\
\hline Cases & 0.0792 & 0.0122 & 0.0708 & 0.0093 & 0.0230 & 0.0110 & 0.0243 & 0.0064 \\
\hline Deaths & 0.0581 & 0.0104 & 0.0569 & 0.0095 & 0.0440 & 0.0103 & 0.0479 & 0.0087 \\
\hline Recoveries & 0.1185 & 0.0240 & 0.111 & 0.0266 & 0.0799 & 0.0192 & 0.0844 & 0.0230 \\
\hline Active & 0.0831 & 0.0173 & 0.0827 & 0.0187 & 0.0306 & 0.0143 & 0.0304 & 0.0168 \\
\hline Pakistan & \multicolumn{4}{|c|}{$70 \%$ of the Series } & \multicolumn{4}{|c|}{$60 \%$ of the Series } \\
\hline Cases & 0.0143 & 0.0090 & 0.0104 & 0.0055 & 0.0186 & 0.0108 & 0.0171 & 0.0062 \\
\hline Deaths & 0.0175 & 0.0081 & 0.0153 & 0.0062 & 0.0333 & 0.0094 & 0.0316 & 0.0079 \\
\hline Recoveries & 0.0396 & 0.0138 & 0.0409 & 0.0135 & 0.0530 & 0.0162 & 0.0475 & 0.0150 \\
\hline Active & 0.0241 & 0.0126 & 0.0233 & 0.0128 & 0.0308 & 0.0132 & 0.0292 & 0.0169 \\
\hline Iran & \multicolumn{4}{|c|}{$90 \%$ of the Series } & \multicolumn{4}{|c|}{$80 \%$ of the Series } \\
\hline Cases & 0.0162 & 0.0035 & 0.0164 & 0.0017 & 0.0151 & 0.0030 & 0.0154 & 0.0016 \\
\hline Deaths & 0.0191 & 0.0025 & 0.0158 & 0.0019 & 0.0073 & 0.0023 & 0.0073 & 0.0016 \\
\hline Recoveries & 0.0537 & 0.0028 & 0.0206 & 0.0015 & 0.0268 & 0.0025 & 0.0178 & 0.0015 \\
\hline Active & 0.0426 & 0.0175 & 0.0316 & 0.0171 & 0.0324 & 0.0138 & 0.0178 & 0.0131 \\
\hline Iran & \multicolumn{4}{|c|}{$70 \%$ of the Series } & \multicolumn{4}{|c|}{$60 \%$ of the Series } \\
\hline Cases & 0.0034 & 0.0020 & 0.0041 & 0.0017 & 0.0050 & 0.0023 & 0.0046 & 0.0012 \\
\hline Deaths & 0.0030 & 0.0017 & 0.0025 & 0.0009 & 0.0040 & 0.0020 & 0.0028 & 0.0011 \\
\hline Recoveries & 0.0037 & 0.0017 & 0.0019 & 0.0011 & 0.0145 & 0.0022 & 0.0054 & 0.0012 \\
\hline Active & 0.0202 & 0.0115 & 0.0142 & 0.0105 & 0.0285 & 0.0132 & 0.0154 & 0.0112 \\
\hline India & \multicolumn{4}{|c|}{$90 \%$ of the Series } & \multicolumn{4}{|c|}{$80 \%$ of the Series } \\
\hline Cases & 0.0594 & 0.0103 & 0.0577 & 0.0040 & 0.0408 & 0.0088 & 0.0333 & 0.0037 \\
\hline Deaths & 0.0463 & 0.0098 & 0.0445 & 0.0097 & 0.0393 & 0.0096 & 0.0393 & 0.0083 \\
\hline Recoveries & 0.0702 & 0.0176 & 0.0634 & 0.0115 & 0.0656 & 0.0167 & 0.0584 & 0.0102 \\
\hline Active & 0.0600 & 0.0131 & 0.0495 & 0.0117 & 0.0431 & 0.0126 & 0.0376 & 0.0108 \\
\hline India & \multicolumn{4}{|c|}{$70 \%$ of the Series } & \multicolumn{4}{|c|}{$60 \%$ of the Series } \\
\hline Cases & 0.0113 & 0.0068 & 0.0071 & 0.0018 & 0.0164 & 0.0074 & 0.0113 & 0.0024 \\
\hline Deaths & 0.0225 & 0.0068 & 0.0213 & 0.0065 & 0.0220 & 0.0080 & 0.0213 & 0.0072 \\
\hline Recoveries & 0.0215 & 0.0095 & 0.0183 & 0.0072 & 0.0388 & 0.0119 & 0.0293 & 0.0087 \\
\hline Active & 0.0198 & 0.0121 & 0.0181 & 0.0090 & 0.0218 & 0.0122 & 0.0204 & 0.0102 \\
\hline
\end{tabular}


Table 2 (on next page)

Results of Ljung Box test (Q-statistic) for diagnostic checking of the proposed models 
1

\begin{tabular}{lcccccc}
\hline \multirow{2}{*}{$\begin{array}{l}\text { BSTS } \\
\text { Model for }\end{array}$} & $\begin{array}{c}c \\
\text { Q- }\end{array}$ & P-Value & $\begin{array}{c}\text { Q- } \\
\text { Statistic }\end{array}$ & Statistic & P-Value & Q-10 \\
Total Cases & 2.297 & 0.807 & 6.478 & 0.774 & 28.205 & 0.560 \\
Deaths & 10.088 & 0.073 & 12.414 & 0.258 & 42.046 & 0.071 \\
Recoveries & 3.108 & 0.683 & 3.750 & 0.958 & 11.894 & 0.999 \\
Active Cases & 7.658 & 0.176 & 11.639 & 0.310 & 32.847 & 0.411 \\
\hline
\end{tabular}

2 


\section{Table 3 (on next page)}

Summary of forecasts and expected required resources after one month (on August 10, 2020) 
1

\begin{tabular}{|c|c|c|c|c|c|}
\hline Item & $\begin{array}{c}\text { Point } \\
\text { Forecast }\end{array}$ & \multicolumn{2}{|c|}{$\begin{array}{c}95 \% \text { Prediction } \\
\text { Interval } \\
\end{array}$} & RMSPE & RMdSPE \\
\hline \multicolumn{6}{|l|}{ Pakistan } \\
\hline No. of expected cases & 333,308 & 275,034 & 391,077 & 0.0953 & 0.0128 \\
\hline No. of expected deaths & 7,187 & 5,978 & 8,390 & 0.0670 & 0.0116 \\
\hline No. of expected recoveries & 279,602 & 208,420 & 345,740 & 0.1033 & 0.0259 \\
\hline No. of active cases & 63,706 & 19,614 & 95,337 & 0.0966 & 0.0196 \\
\hline \multicolumn{6}{|l|}{ Iran } \\
\hline No. of expected cases & 325,391 & 283,403 & 369,613 & 0.0176 & 0.0035 \\
\hline No. of expected deaths & 17,458 & 15,538 & 19,476 & 0.0370 & 0.0026 \\
\hline No. of expected recoveries & 294,493 & 243,470 & 350,203 & 0.0227 & 0.0023 \\
\hline No. of active cases & 5,892 & 973 & 73,416 & 0.0462 & 0.0183 \\
\hline \multicolumn{6}{|l|}{ India } \\
\hline No. of expected cases & $1,874,037$ & $1,538,452$ & $2,393,316$ & 0.0690 & 0.0132 \\
\hline No. of expected deaths & 39,464 & 30,407 & 48,765 & 0.0554 & 0.0098 \\
\hline No. of expected recoveries & $1,299,328$ & $1,091,762$ & $1,618,996$ & 0.0790 & 0.0236 \\
\hline No. of active cases & 517,153 & 390,286 & 717,715 & 0.0665 & 0.0167 \\
\hline
\end{tabular}
2 
Figure 1

Current Trends of the outbreak

The data points represent the ratios for different parameters of the pandemic. The green line indicates the Ratio of cumulative number of cases to cumulative number of tests (RCT). Blue line indicates the ratio of cumulative number of deaths to the cumulative number of cases (RDC). The red line shows the ratio of cumulative number of recoveries to the cumulative number of cases ( $R R C)$. The yellow line represents the ratio of cumulative number of active cases to the cumulative number of confirmed cases ( RAC). 


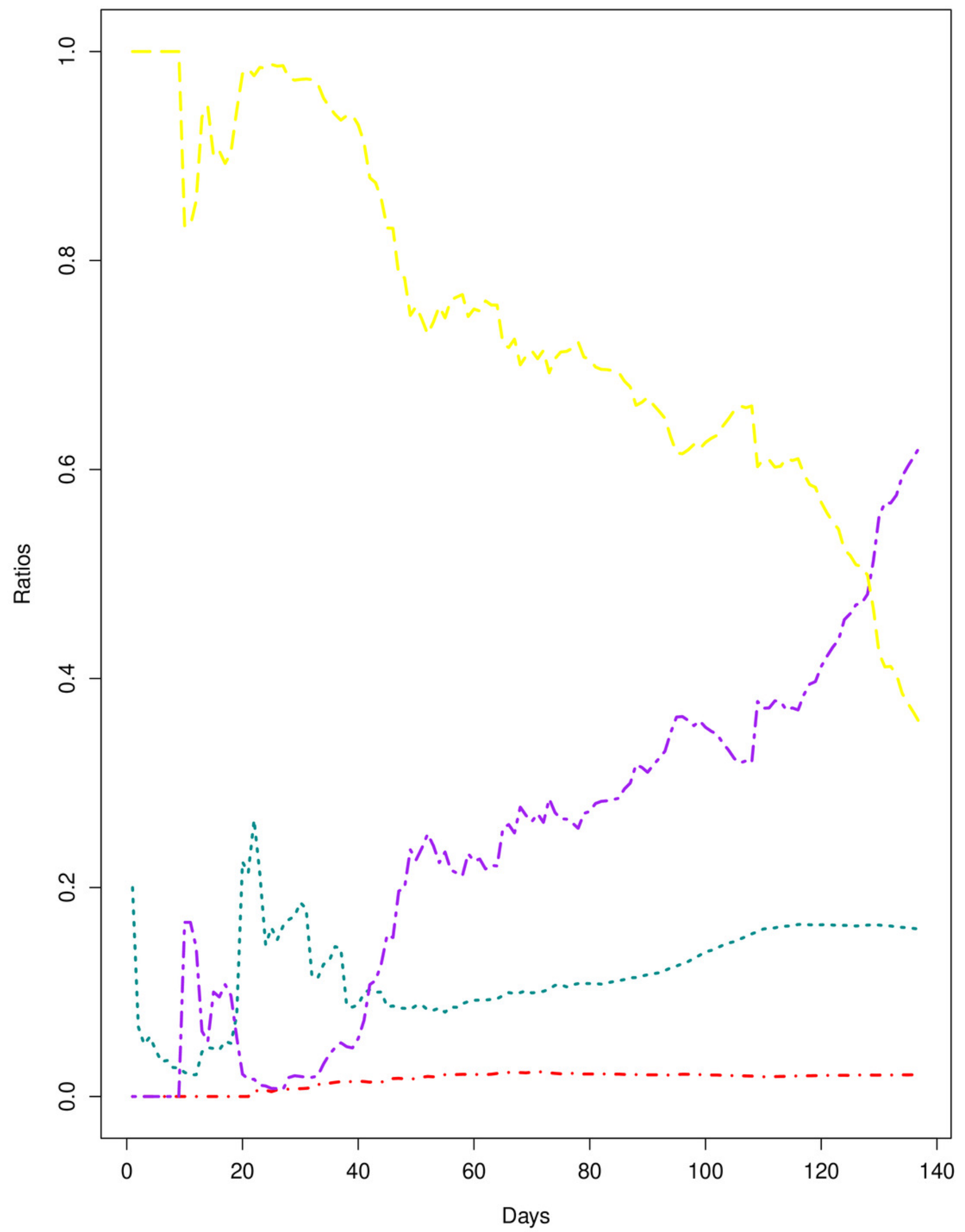

PeerJ reviewing PDF | (2020:07:50949:2:1:NEW 1 May 2021) 


\section{Figure 2}

Forecasts for different parameters of the pandemic using BSTS models

Each line represents the observed versus fitted and forecast values. The light green represent the forecasts for cumulative number of cases. The dashed dark green line shows the fitted values for cumulative number of cases (which have overlapped the observed numbers of cases given in light green color). The blue line indicates the forecasts for the cumulative number of deaths. The light blue (dotted/dashed) line represents the fitted values for the cumulative number of deaths (which have overlapped the observed numbers of deaths presented in blue color). The light purple line shows the forecasts for the cumulative number of recoveries. The purple line (dotted) depicts the fitted values for the recoveries (which have overlapped the observed numbers of recoveries presented in light purple color). The light maroon line shows the cumulative number of active cases. The dark maroon line represents the fitted values for the active cases (which have overlapped the observed numbers of active cases presented in light maroon color) 


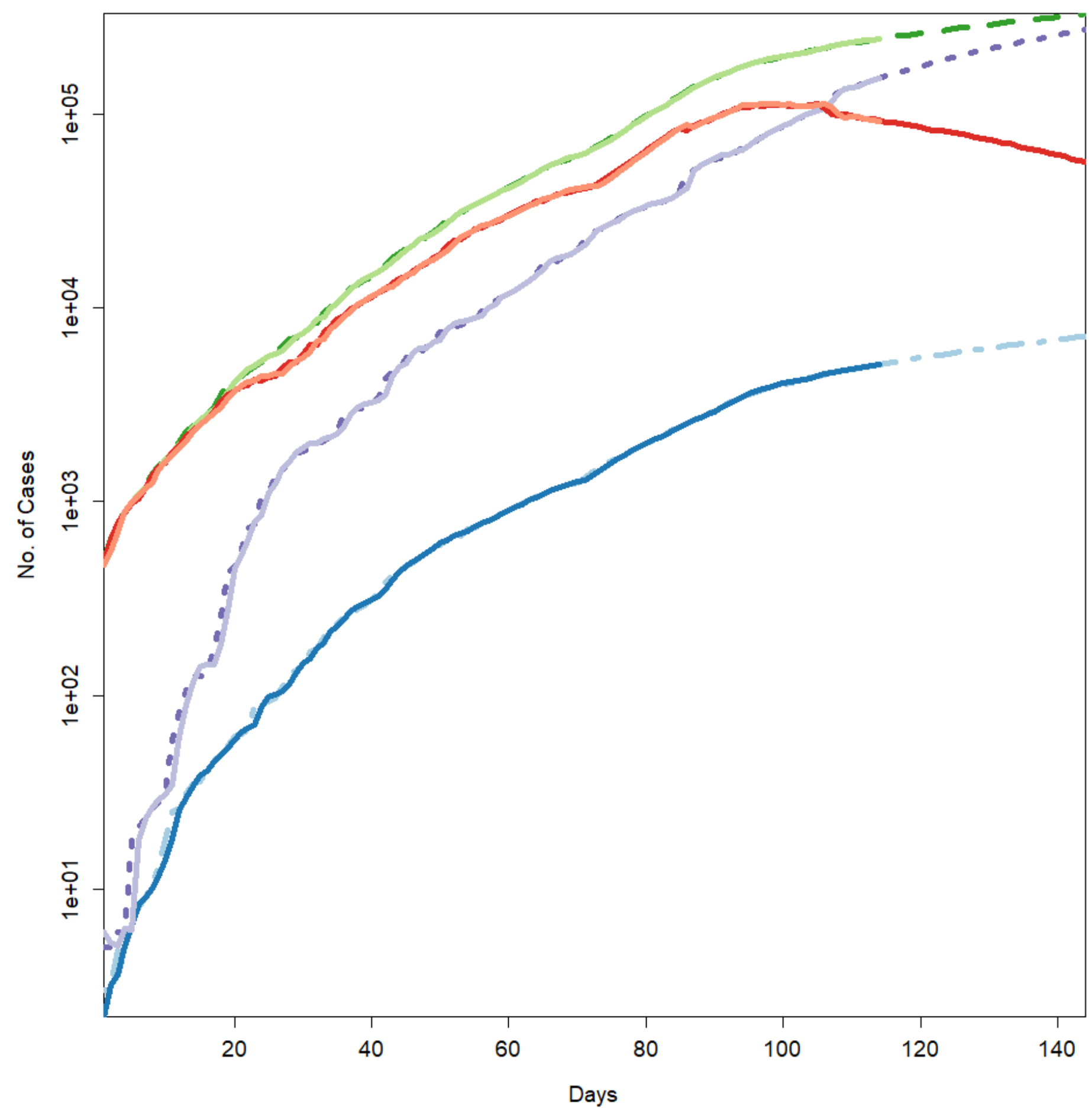


Figure 3

Analysis of trend, seasonality and trend components

The panel (A) shows the contribution of trend component. The panel (B) shows the contribution of seasonality and the panel (C) shows the contribution of regression component of the series. 

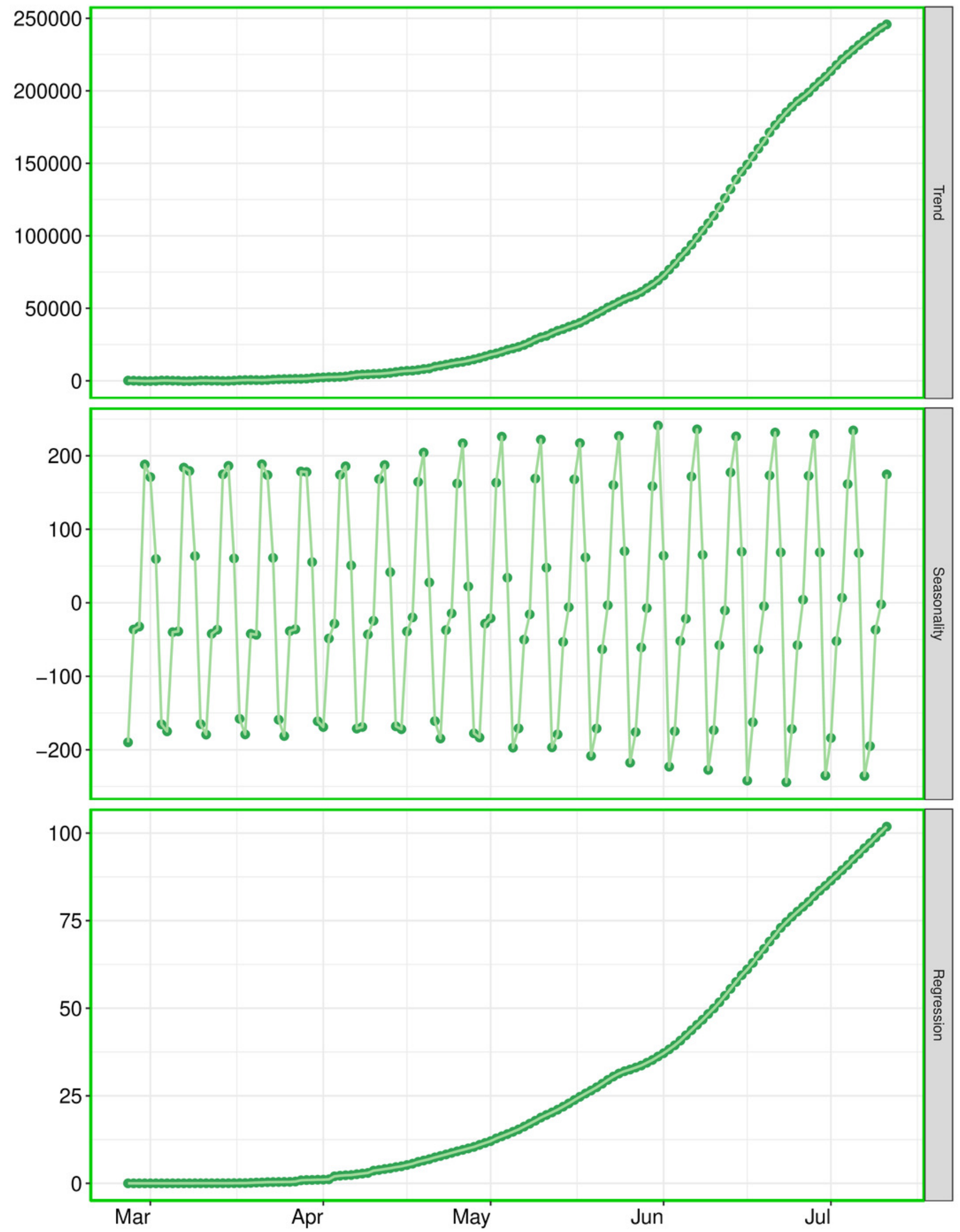


\section{Figure 4}

Analysis of causal impacts of lifting lockdown in the country

The data points indicate the observed and expected number (hand the lockdown not lifted) of cumulative cases. The black line shows the observed number of cases. The grey line with grey shade indicates the expected number of cases, had the lockdown not lifted. 


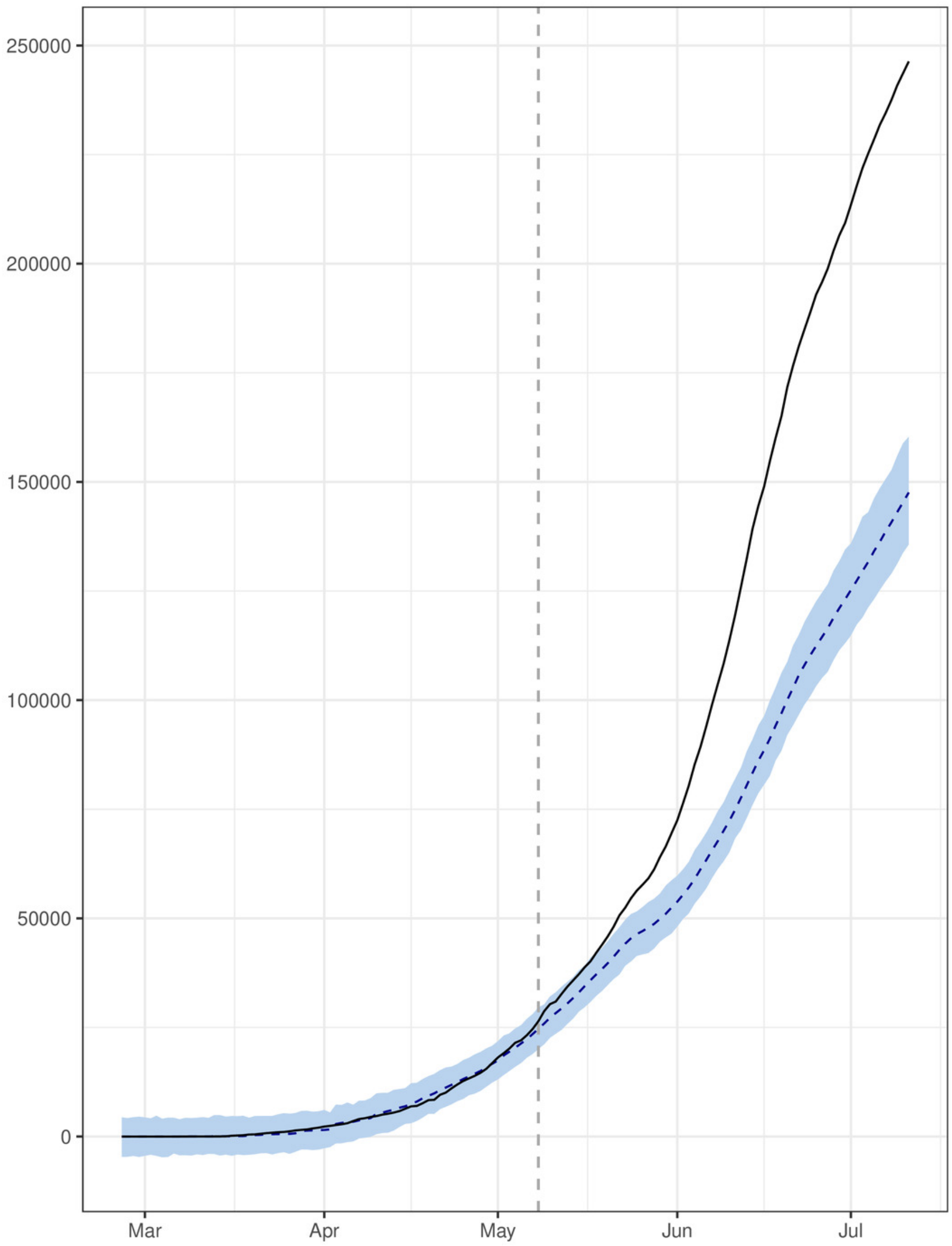


Figure 5

Trace plots for one-step-ahead prediction error variance (PEV) using MCMC chains for cumulative number of cases in Pakistan

The blue lines show the average of MCMC draws at each iteration 


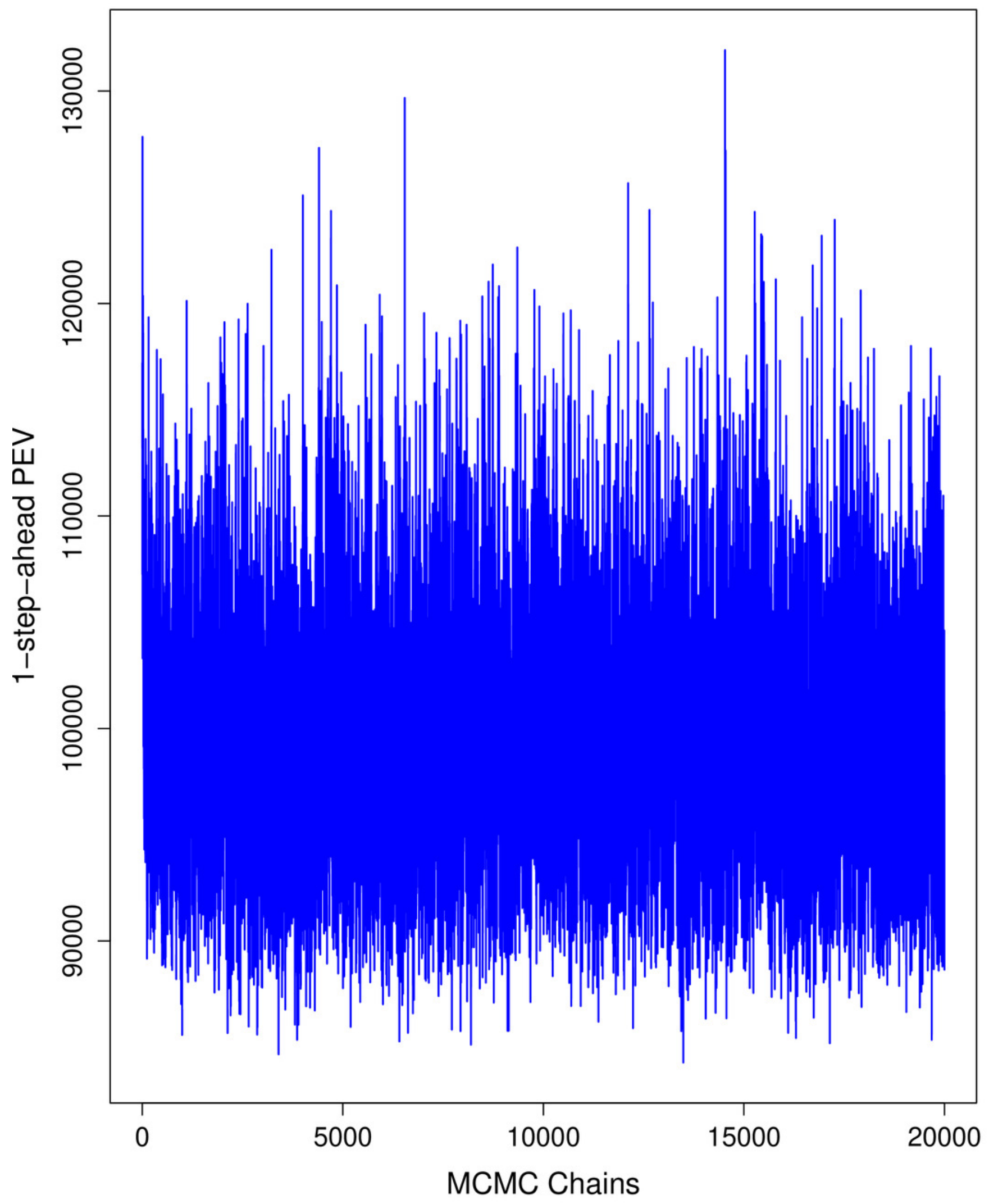


Figure 6

Trace plots for one-step-ahead prediction error variance (PEV) using MCMC chains for cumulative number of deaths in Pakistan

The blue lines show the average of MCMC draws at each iteration. 


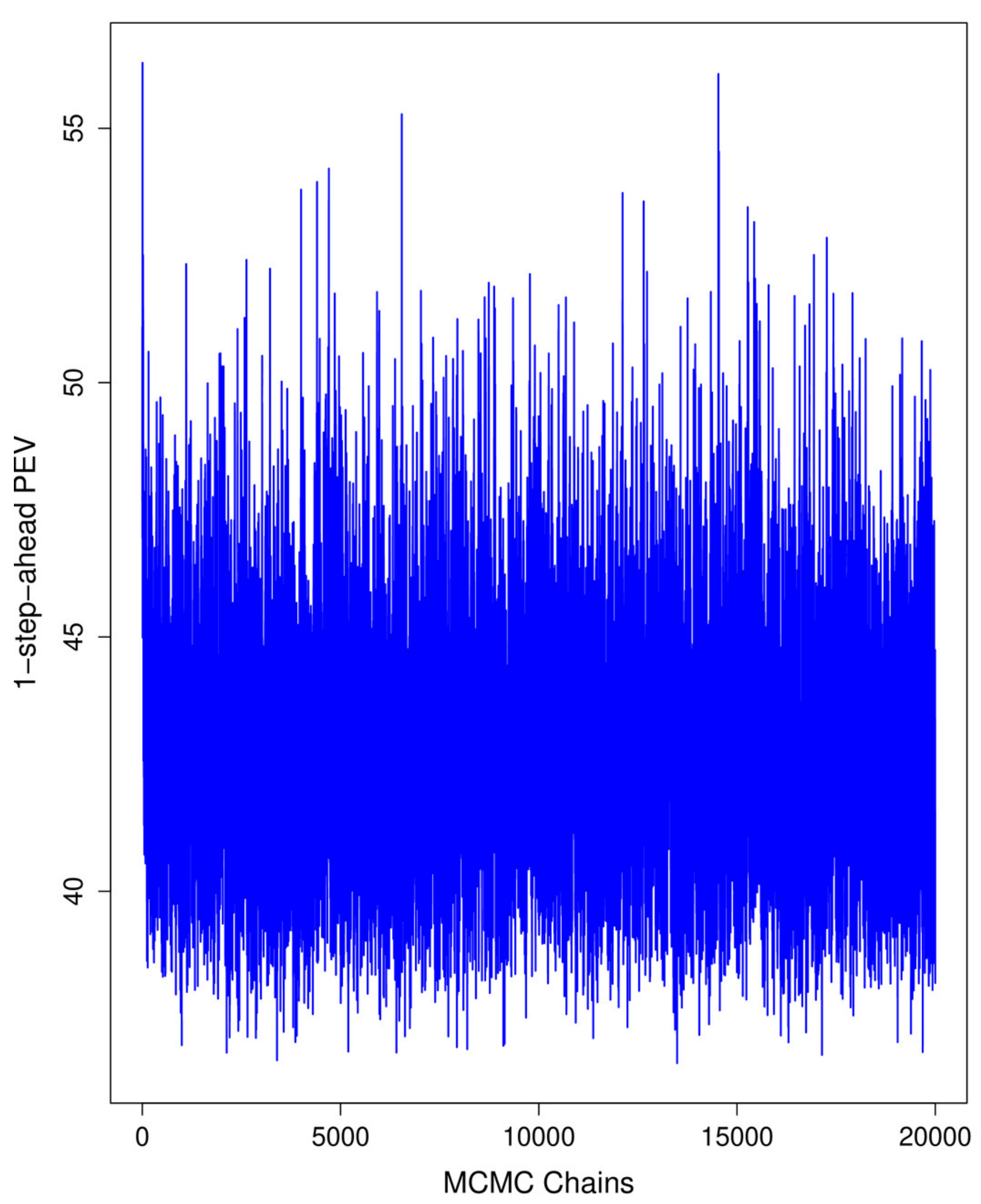


Figure 7

Trace plots for one-step-ahead prediction error variance (PEV) using MCMC chains for cumulative number of recoveries in Pakistan

The blue lines show the average of MCMC draws at each iteration 


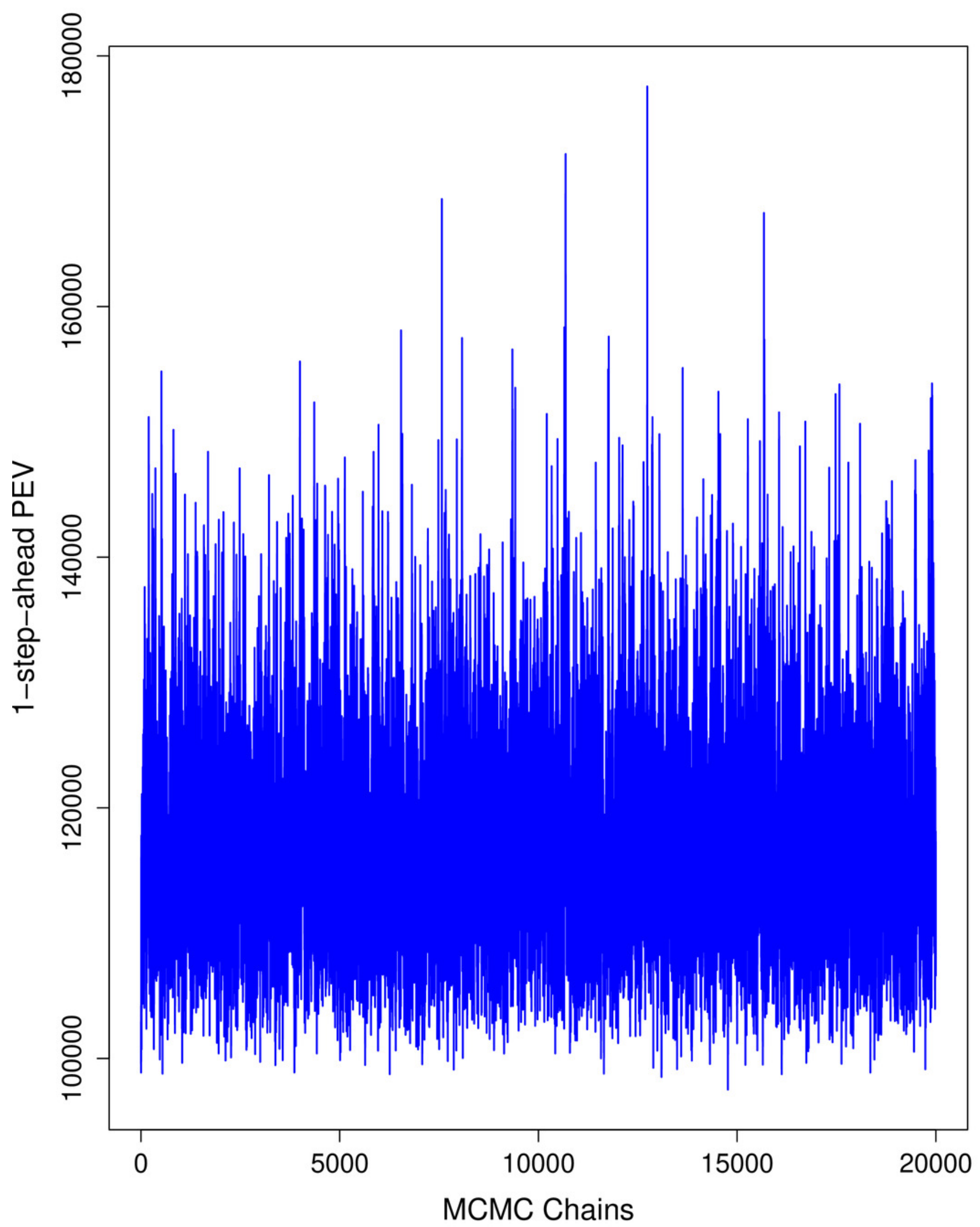


Figure 8

Trace plots for one-step-ahead prediction error variance (PEV) using MCMC chains for number of active cases in Pakistan

The blue lines show the average of MCMC draws at each iteration 


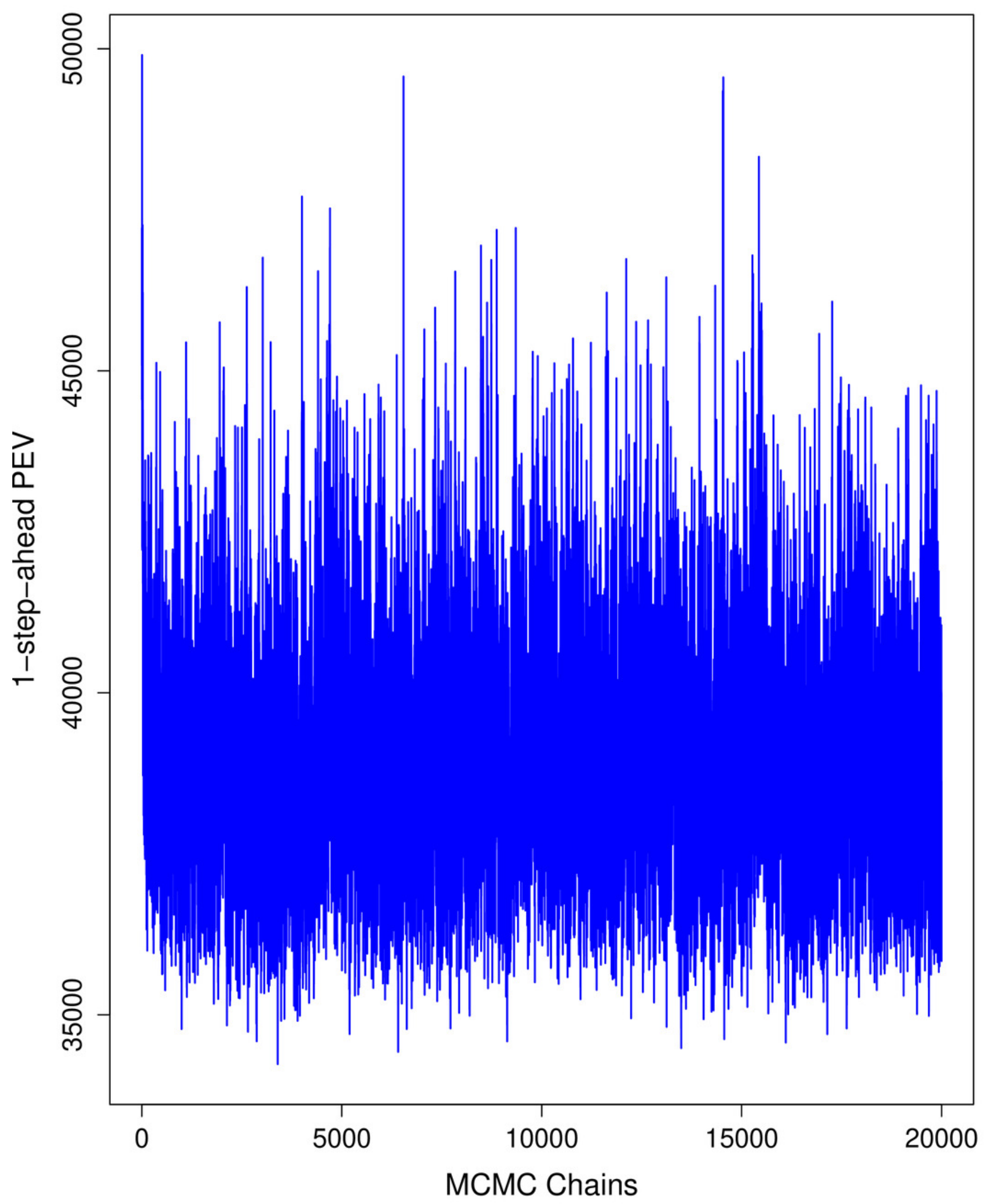


Figure 9

QQ plots for the residual terms for cumulative number of cases in Pakistan

The black line is the reference line for the standard normal distribution and means of MCMC draws have been represented by blue dots. 


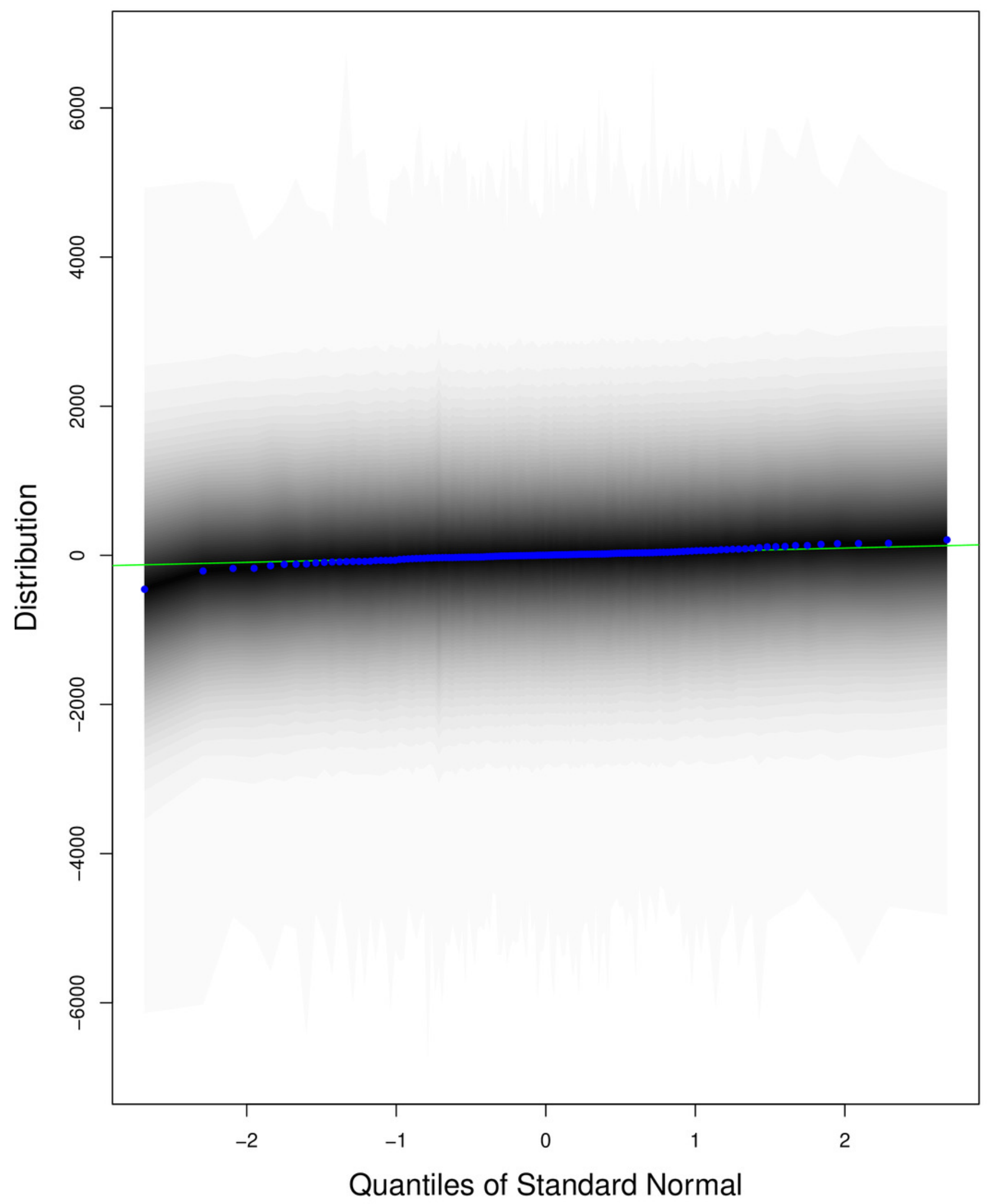




\section{Figure 10}

QQ plots for the residual terms for cumulative number of deaths in Pakistan

The black line is the reference line for the standard normal distribution and means of MCMC draws have been represented by blue dots. 


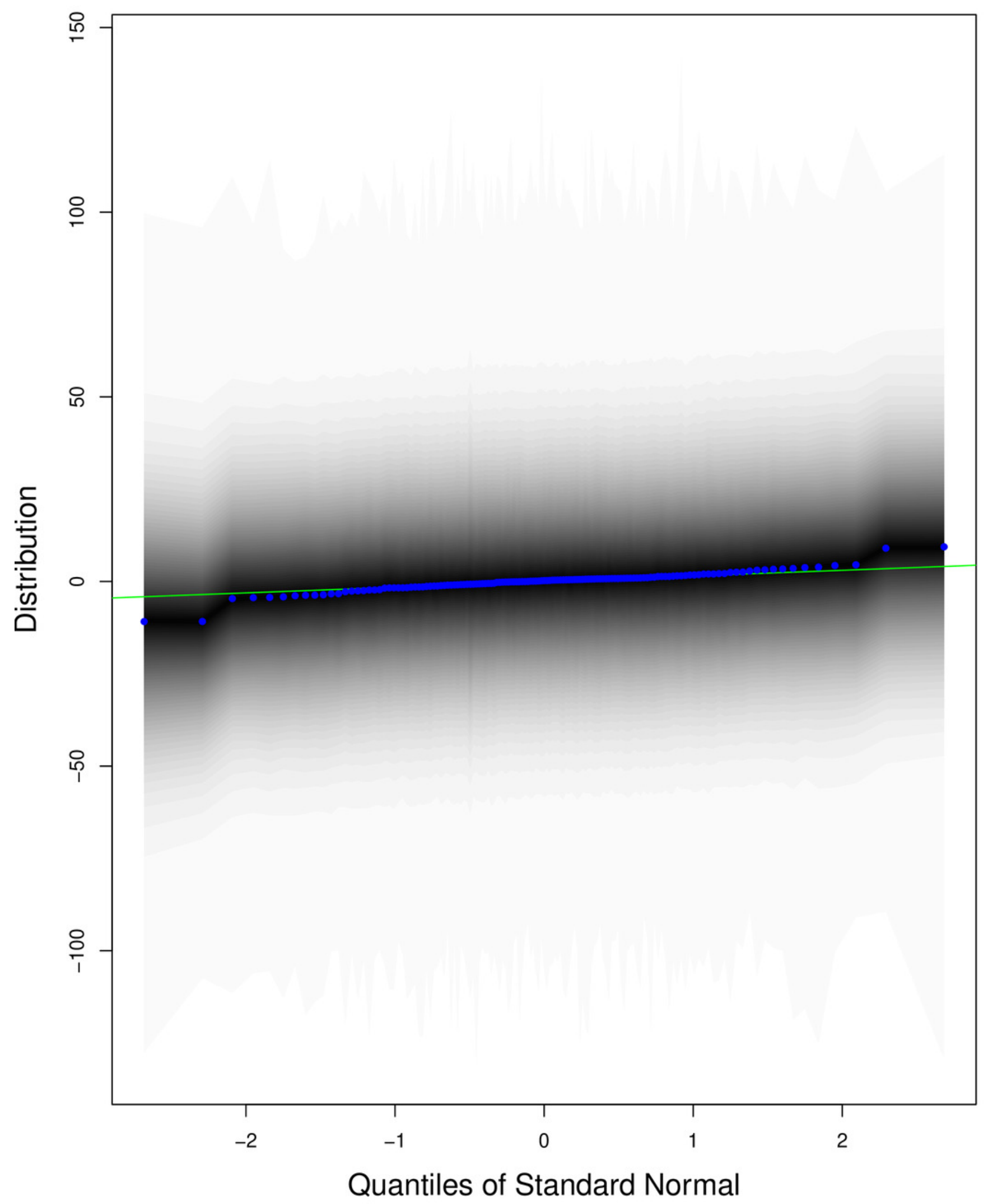


Figure 11

QQ plots for the residual terms for cumulative number of recoveries in Pakistan

The black line is the reference line for the standard normal distribution and means of MCMC draws have been represented by blue dots. 


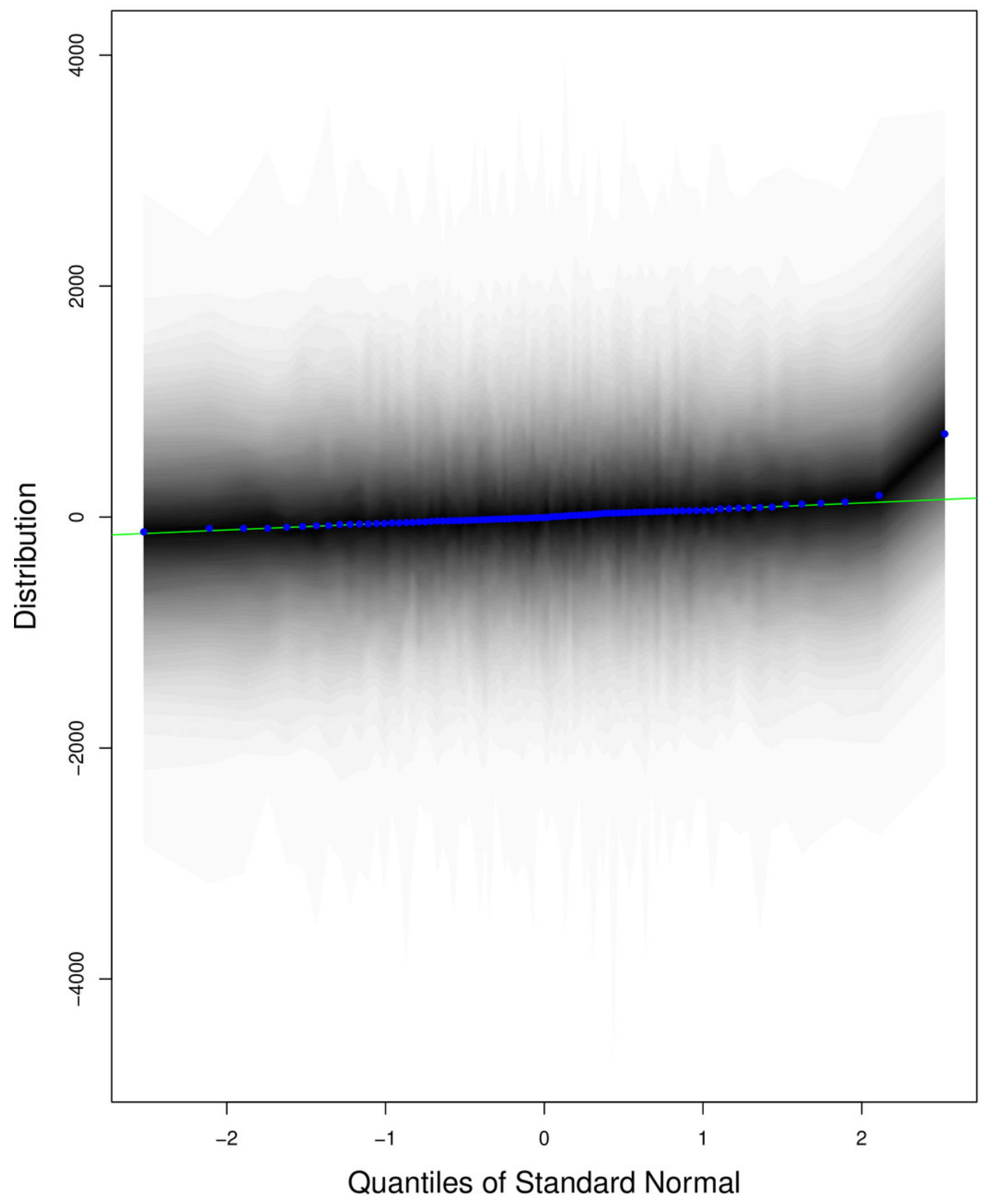


Figure 12

QQ plots for the residual terms for number of active cases in Pakistan

The black line is the reference line for the standard normal distribution and means of MCMC draws have been represented by blue dots. 


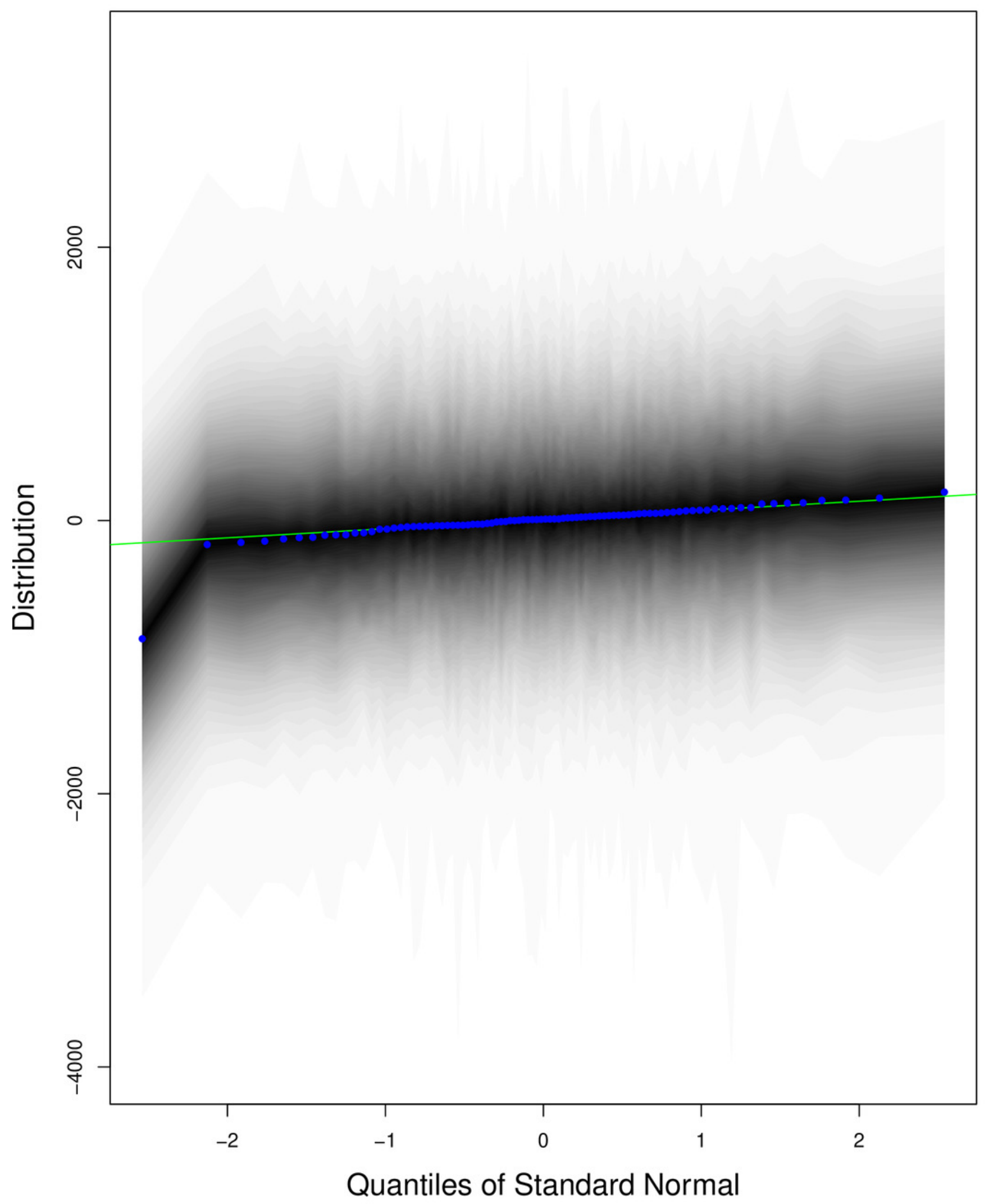


Figure 13

Posterior distribution of the autocorrelation function (ACF) of residuals using boxplots for cumulative number of cases in Pakistan

The blue boxes represent side-by-side box plots 


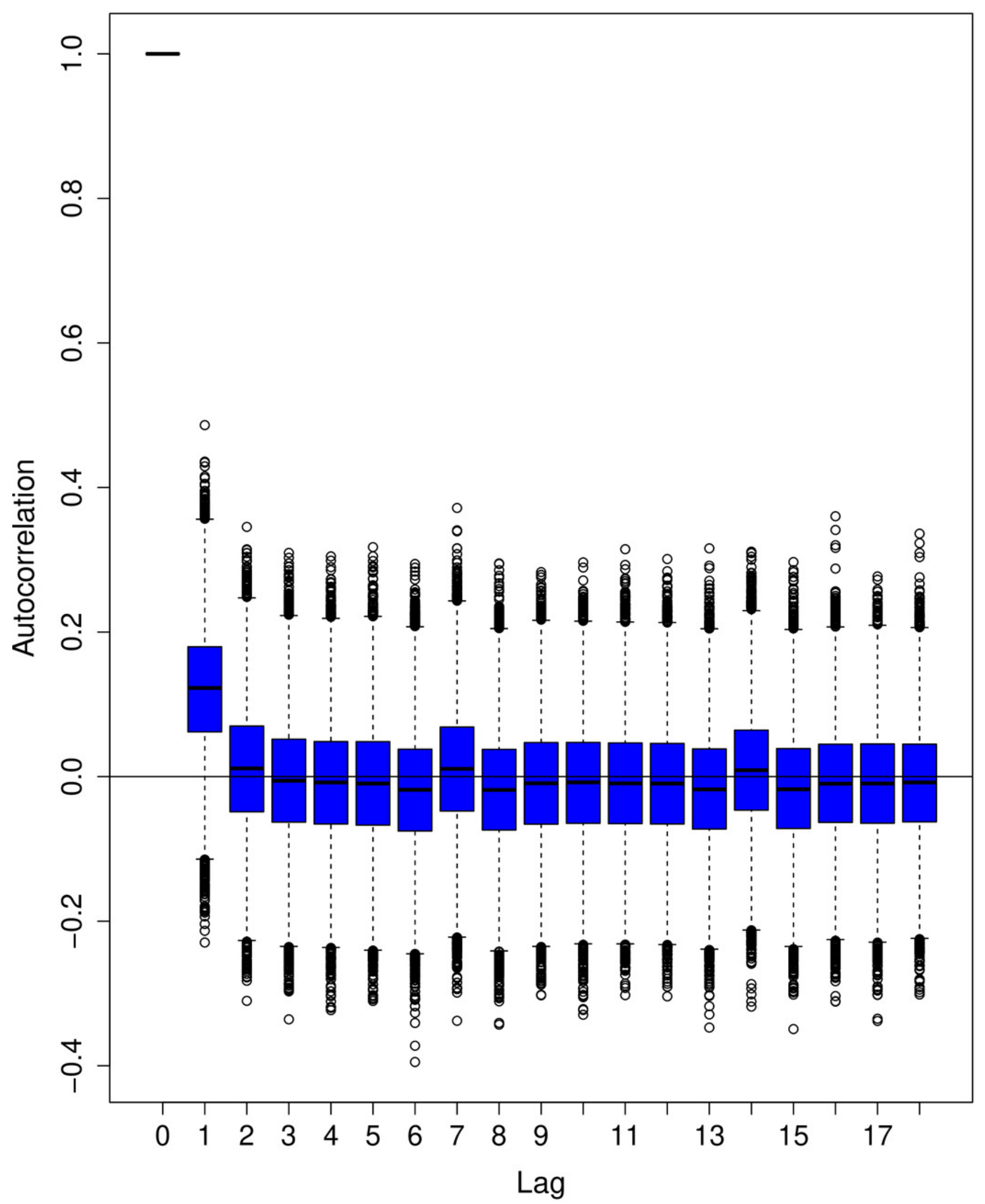


Figure 14

Posterior distribution of the autocorrelation function (ACF) of residuals using boxplots for cumulative number of deaths in Pakistan

The blue boxes represent side-by-side box plots 


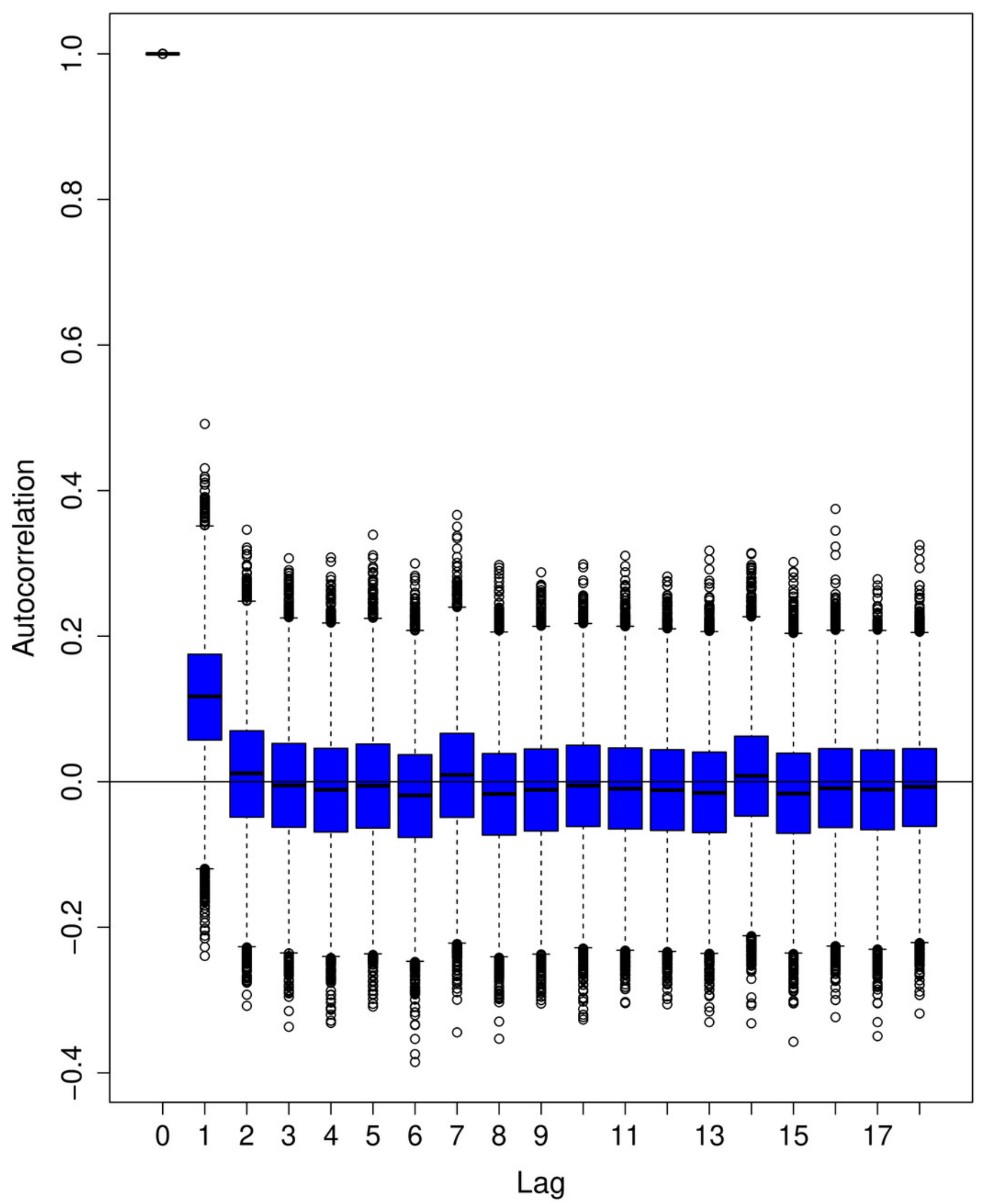


Figure 15

Posterior distribution of the autocorrelation function (ACF) of residuals using boxplots for cumulative number of recoveries in Pakistan

The blue boxes represent side-by-side box plots 


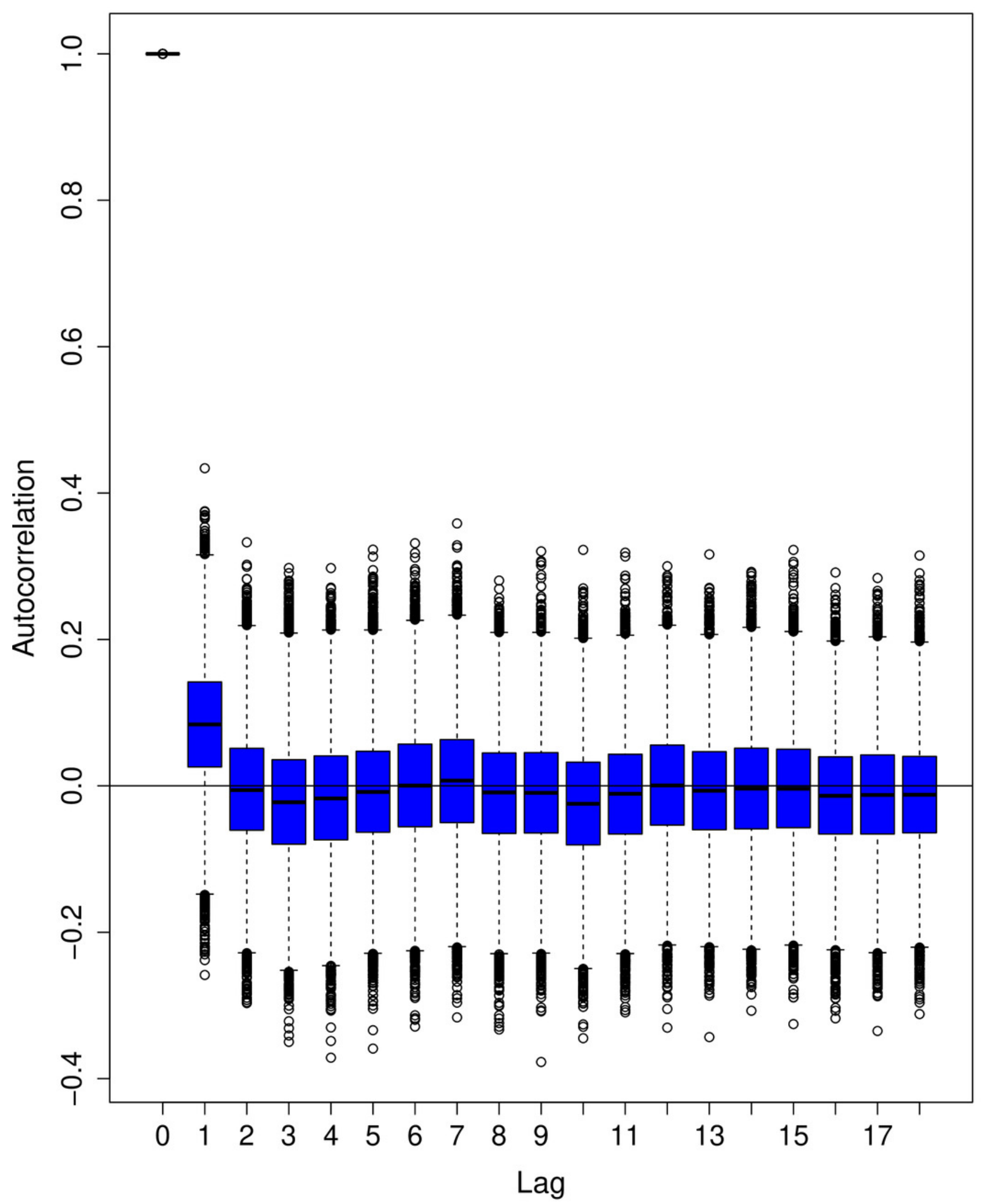


Figure 16

Posterior distribution of the autocorrelation function (ACF) of residuals using boxplots for number of active cases in Pakistan

The blue boxes represent side-by-side box plots 


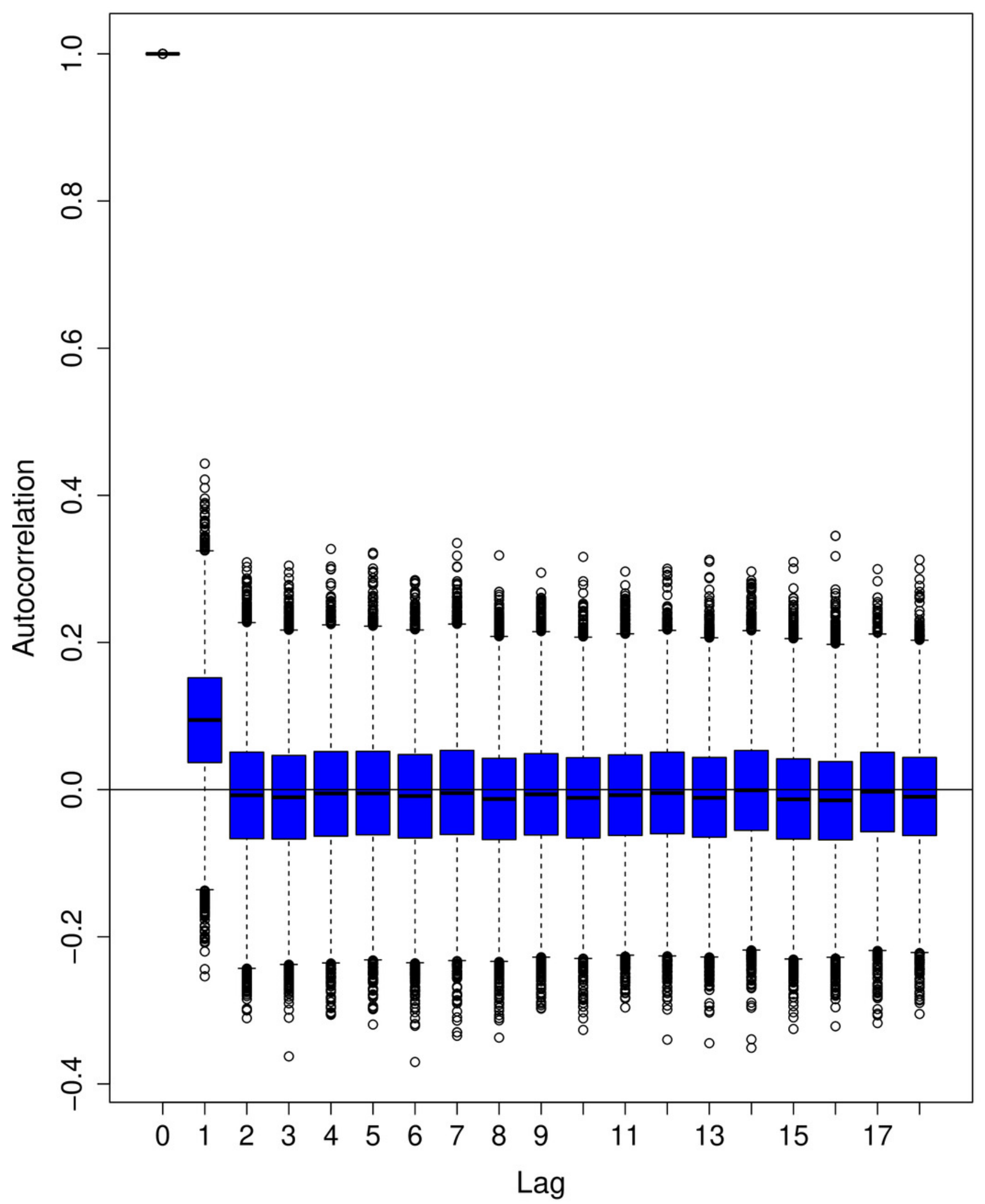




\section{Figure 17}

Comparison of true versus fitted series using $90 \%$ of the true dataset

Black line shows the actual series. Maroon line shows fitted series and shaded (light maroon) area shows the $95 \%$ prediction interval. 


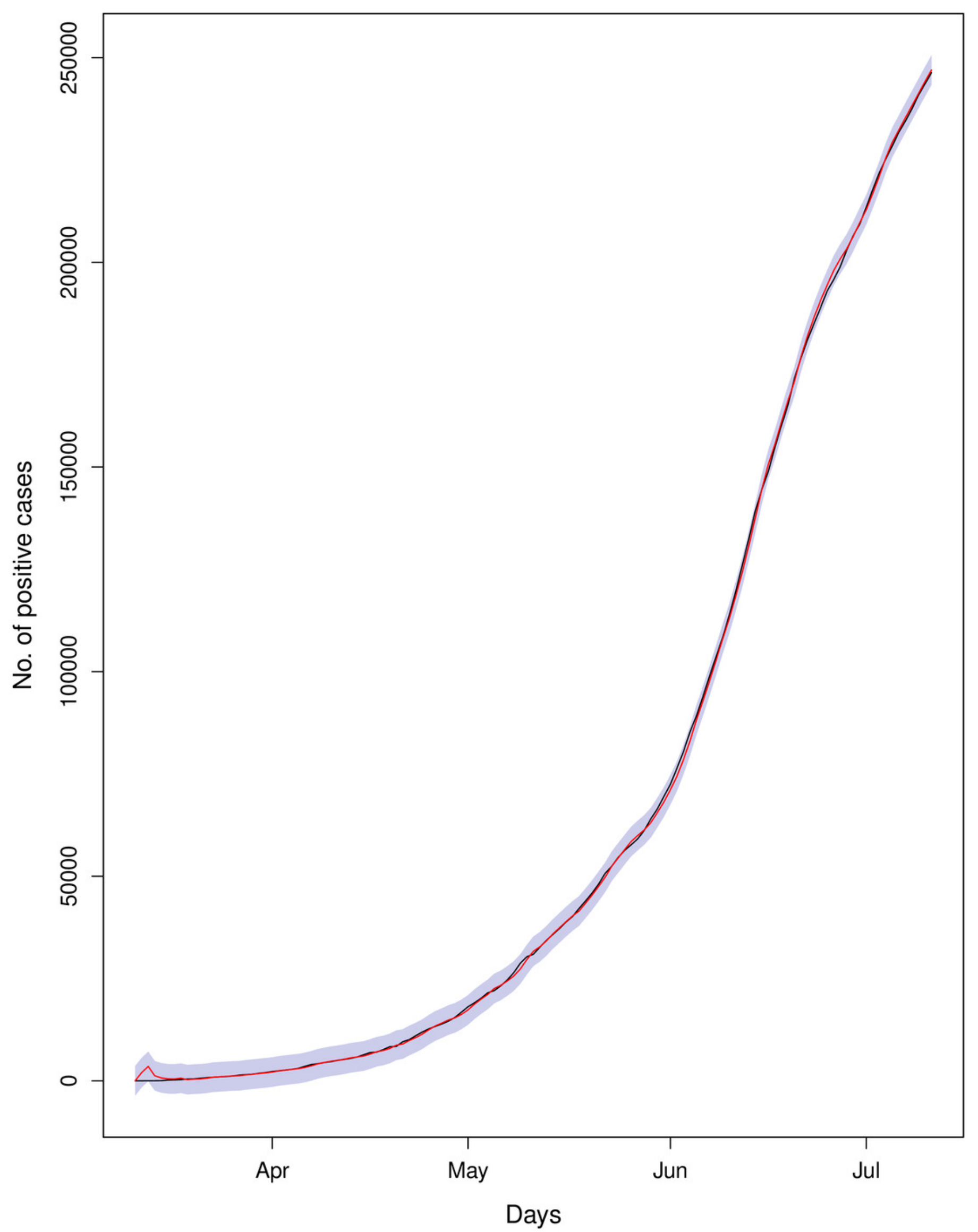

PeerJ reviewing PDF | (2020:07:50949:2:1:NEW 1 May 2021) 


\section{Figure 18}

Comparison of true versus fitted series using $80 \%$ of the true dataset

Black line shows the actual series. Maroon line shows fitted series and shaded (light maroon) area shows the $95 \%$ prediction interval. 


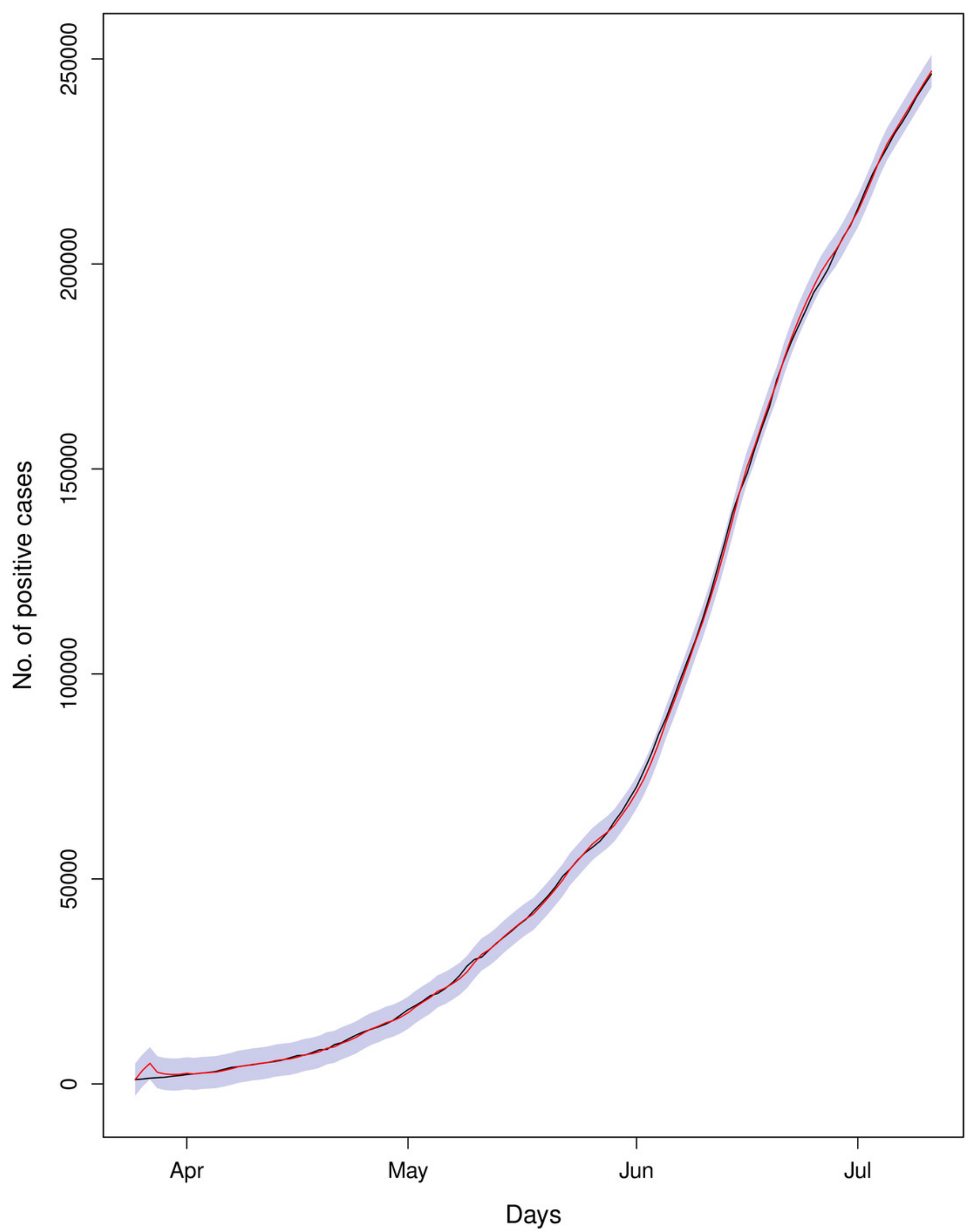

PeerJ reviewing PDF | (2020:07:50949:2:1:NEW 1 May 2021) 


\section{Figure 19}

Comparison of true versus fitted series using $70 \%$ of the true dataset

Black line shows the actual series. Maroon line shows fitted series and shaded (light maroon) area shows the $95 \%$ prediction interval. 


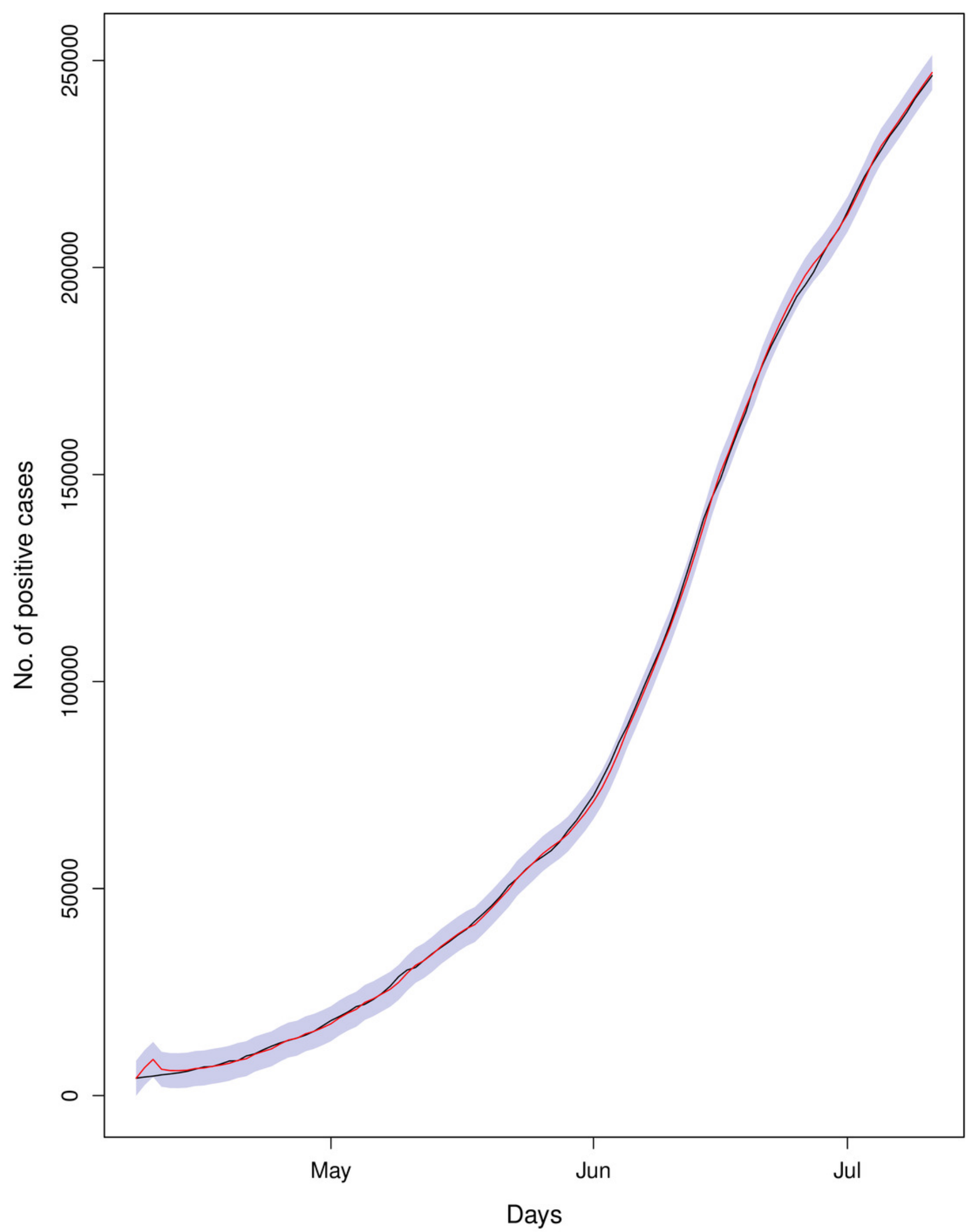

PeerJ reviewing PDF | (2020:07:50949:2:1:NEW 1 May 2021) 
Figure 20

Comparison of true versus fitted series using $60 \%$ of the true dataset

Black line shows the actual series. Maroon line shows fitted series and shaded (light maroon) area shows the $95 \%$ prediction interval. 


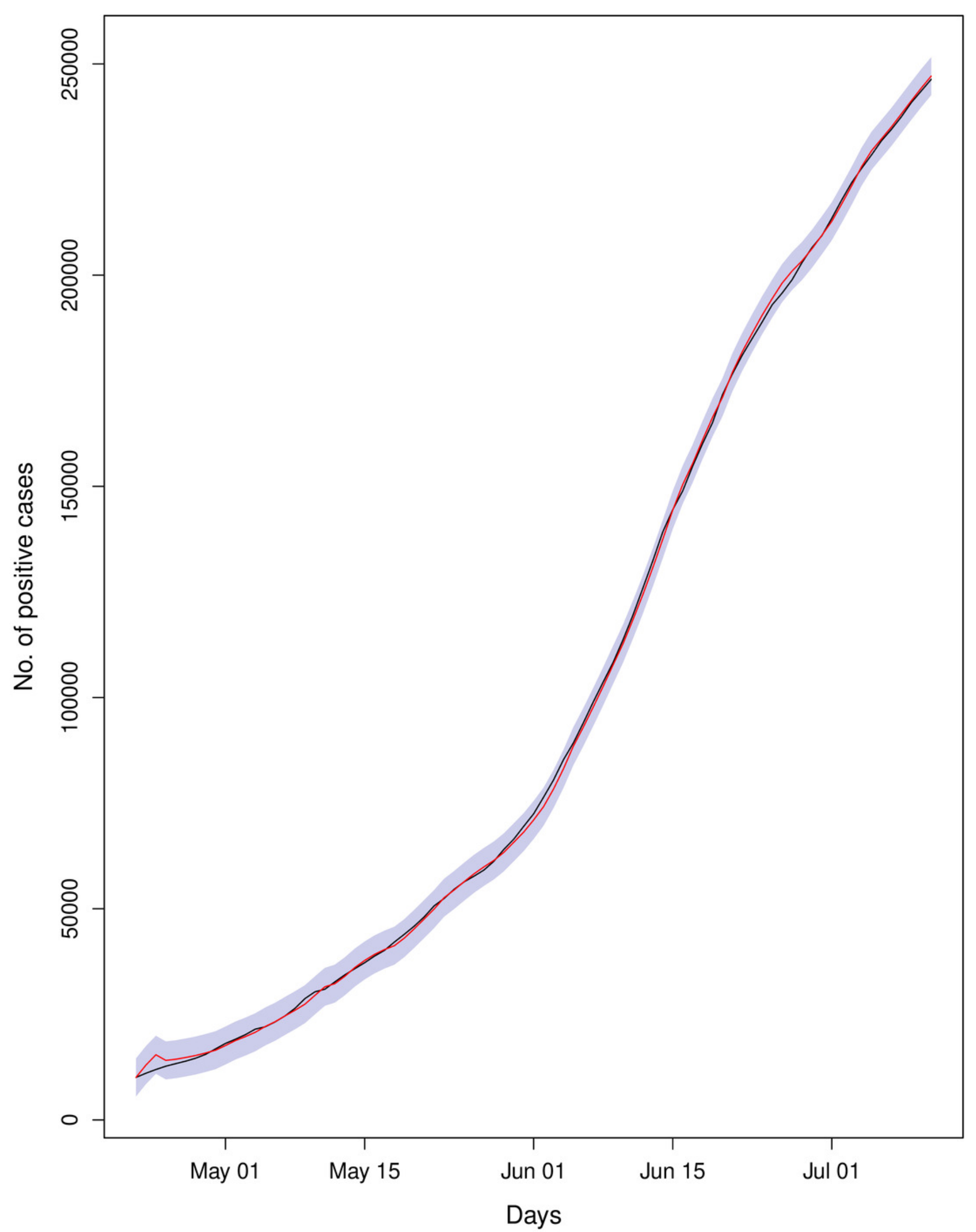

PeerJ reviewing PDF | (2020:07:50949:2:1:NEW 1 May 2021) 
Figure 21

Comparison of true versus fitted series using $50 \%$ of the true dataset

Black line shows the actual series. Maroon line shows fitted series and shaded (light maroon) area shows the $95 \%$ prediction interval. 


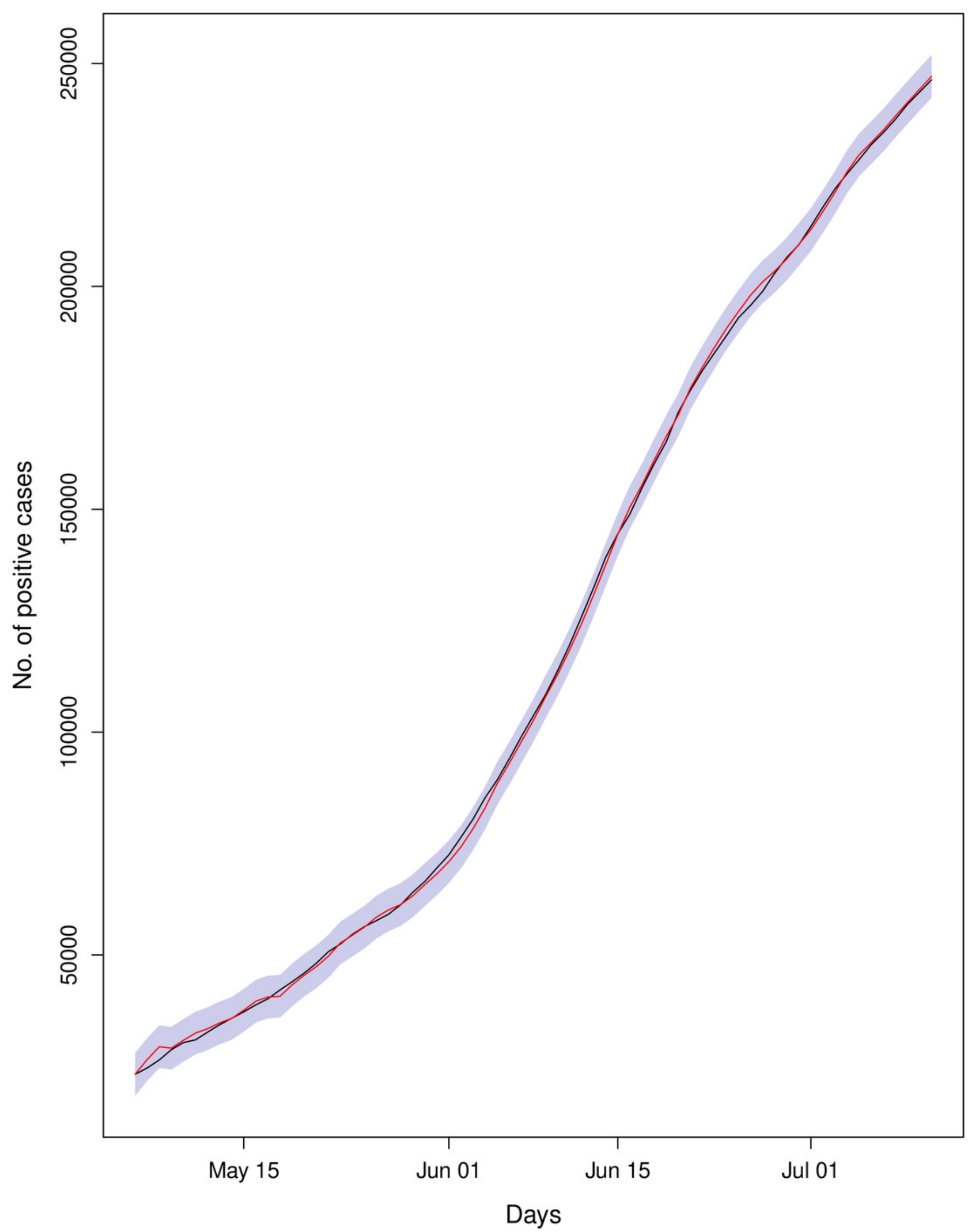

PeerJ reviewing PDF | (2020:07:50949:2:1:NEW 1 May 2021) 